\title{
Cumulant Structure Factors of Jellium
}

\author{
P. Ziesche' ${ }^{1}$, J. Cioslowski ${ }^{1,2}$ \\ ${ }^{1}$ Max-Planck-Institut für Physik Komplexer Systeme, Dresden, Germany \\ ${ }^{2}$ Institute of Physics, University of Szczecin, Szczecin, Poland \\ Email:pz@pks.mpg.de
}

Received 29 January 2014; revised 25 February 2014; accepted 21 March 2014

Copyright (C) 2014 by authors and Scientific Research Publishing Inc.

This work is licensed under the Creative Commons Attribution International License (CC BY).

http://creativecommons.org/licenses/by/4.0/

(c) (i) Open Access

\section{Abstract}

For the ground state of the homogeneous electron gas (jellium), it is shown how the cumulant decomposition of the 2-matrix leads to the cumulant decomposition of the structure factors $S_{\mathrm{a}, \mathrm{p}}(q)$ for the antiparallel (a) and parallel (p) spin pairs and how it simultaneously allows one to derive the momentum distribution $n(k)$, which is a one-body quantity [Phys. Rev. A 86, 012508 (2012), A 89, 059902 (E)(2014)]. The small- $q$ and large- $q$ behavior of $S_{\mathrm{a}, \mathrm{p}}(q)$, and their normalizations are derived and compared with the results of P. Gori-Giorgi et al. [Physica A 280, 199 (2000) and Phys. Rev. B 61, 7353 (2000)].

\section{Keywords}

Jellium, Reduced Density Matrices, Pair Densities, Coulomb Hole, Fermi Hole, Structure Factors, Cumulant Decomposition

\section{Introduction}

This paper deals with the ground state of an extended spin-unpolarized (paramagnetic) homogeneous electron gas (HEG), which is one of the most widely studied systems with correlated electrons [1]. The gas is assumed to be uniform in space with the electron density $\rho \sim r_{s}^{-3}$. Other characteristic quantities that depend parametrically on $r_{s}$ are the total energy per electron $e$, from which follows the interaction energy $v=r_{s} \mathrm{de} / \mathrm{d} r_{s}$ [2], the momentum distribution $n(k)$, and the static structure factors $(\mathrm{SFs}) S_{\mathrm{a}, \mathrm{p}}(q)$ with "a" standing for electron pairs with antiparallel spins and "p" for pairs with parallel spins. For recent papers on the HEG, also known as jellium, see for example [3]-[15]. Recent GWA and QMC calculations for $n(k)$ are [3] [4]. (For comparison with experiment see [5]. The value of $z_{\mathrm{F}} \approx 0.6$ for $r_{s}=3.9$ agrees with the results, obtained in Refs. [6] [7].)

Overhauser considered the pair densities PDs $g_{\mathrm{a}, \mathrm{p}}(r)$, which follow from the SFs by Fourier transform, and parameterized them in terms of certain 2-body wave functions (known as geminals) [16]. More general geminals should allow one to calculate not only the 2-body quantities $S_{\mathrm{a}, \mathrm{p}}(q)$, but also the 1-body quantity $n(k)$. 
Searching in this direction, one immediately encounters the hierarchy of the reduced density matrices (RDMs) and their cumulant decomposition. (The concept of RDM is of great importance in quantum chemistry, see [17][22].) Here it is assumed that the 2-body RDM (2-matrix) is available from perturbation theory or otherwise (hierarchy truncation etc.) and, with the spin structure as a decisive point, it is elucidated how the contraction has to be performed for an extended system in such a way that the 1-body density $n(k)$ is obtained. In view of the thermodynamic limit, this procedure calls for the cumulant decomposition of the 2-matrix into its HartreeFock part $\gamma_{\mathrm{HF}}$ and its nonreducible remainder, the cumulant 2-matrix $\gamma_{\mathrm{c}}$, which proves to be the source of both $n(k)$ and $S_{\mathrm{a}, \mathrm{p}}(q)$ [10]. Diagonalization of $\gamma_{\mathrm{c}}$ yields cumulant geminals and the corresponding weights (i.e. the spectral resolution).

A side product of this geminal analysis is the following. Usually the partitioning $S_{\mathrm{p}}=S_{0}+\Delta S_{\mathrm{p}}$ involves an unperturbed (ideal Fermi gas) term $S_{0}$ and the interaction-induced term $\Delta S_{\mathrm{p}}$ arising from the pairwise Coulomb repulsion [15]. In contrast to that approach, an alternative partitioning is presented here as a consequence of the cumulant decomposition of the 2-matrix, namely $S_{\mathrm{p}}=S_{\mathrm{HF}}-C_{\mathrm{p}}$, with the Hartree-Fock term $S_{\mathrm{HF}}$ [constructed from the correlated $n(k)$ ] and a nonreducible remainder, i.e. the cumulant parallel-spin SF $C_{\mathrm{p}}$. The advantage of such a partitioning stems from the fact that the cumulant SFs $C_{\mathrm{a}, \mathrm{p}}$ are given within perturbation theory by linked (and therefore size-extensive) diagrams with two open particle-hole lines and arbitrary numbers of closed loops. As usual, the divergent terms are eliminated by a partial summation known as "the random phase approximation" (RPA) with the physical meaning of long-range correlations (screening), which becomes exact at the high-density limit of $r_{s} \rightarrow 0$ [23] [24]. RPA is a general method to treat the quantum-mechanical many-body problem, successfully applied not only to the HEG. It is now implemented in electronic structure codes for both solids and molecules [25]. A recent discussion concerns its failure when applied to the dissociation of $\mathrm{H}_{2}$ : it is exact at the dissociation limit, but very inaccurate at intermediate internuclear distances, see Figure 1 in [26]. This RPA has been applied to the HEG quantities $e, v, n(k)$, and $S_{\mathrm{a}, \mathrm{p}}(q)$. There have been recent efforts to obtain $n(k)$ and $S(q)$ beyond RPA [7] [15]. In those publications, analytical constraints and fitting procedures to quantum-Monte-Carlo data (as "amends" for experimental data) have been combined for the best possible results. Other attempts to go beyond RPA and quantum-Monte-Carlo calculations are the operatorproduct expansion (OPE) [27] and the machine learning methods [28]. In the following text, the RPA is combined with the cumulant analysis described above. Altogether, the available data on the static SFs $S_{\mathrm{a}, \mathrm{p}}(q)$ of the HEG are compiled and analyzed from a new viewpoint, namely the cumulant decomposition, continuing earlier attempts in that direction [7] [10] [29]-[32]. From a formal point of view, the present study is closely related to the work of P. Gori-Giorgi et al. [15].

The paper is organized as follows. In Section 2, the 2-matrix $\gamma_{2}$ and its cumulant decomposition is introduced. It is shown how the SFs $C_{\mathrm{a}, \mathrm{p}}(q)$ and $S_{\mathrm{a}, \mathrm{p}}(q)$ follow from $\gamma_{2}$. Their relation to the PDs $g_{\mathrm{a}, \mathrm{p}}(r)$ via the Fourier transform is elucidated in Appendix A, where the cusp and curvature theorems are summarized as well. In Section 3, the antiparallel-spin SFs are derived within RPA [the necessary technical details (particlehole propagator, etc.) being given in Appendix B]. The normalization of $S_{\mathrm{a}}(q)$ yields the short-range correlation parameter $g_{\mathrm{a}}(0)$. In Section 4, the cumulant decomposition of the parallel-spin SFs with a complicated exchange integral is presented. The fourth moment of $S_{p}(q)$ yields another short-range correlation parameter, namely $g_{\mathrm{p}}^{\prime \prime}(0)$. Table 1 and Table 2 summarize the small- and large- $q$ behavior and the normalization of the SFs. Section 5 concludes the paper with a summary and an outlook.

\section{Basic Equations}

The functions mentioned above, i.e. $n(k)$ and $S(q)=S_{\mathrm{a}}(q)+S_{\mathrm{p}}(q)$, determine the total energy $e=t+v$ via the kinetic energy $t$ and the interaction energy $v$,

$$
t=\int_{0}^{\infty} \mathrm{d}\left(k^{3}\right) n(k) \frac{k^{2}}{2}, \quad v=-\frac{1}{4} \int_{0}^{\infty} \mathrm{d}\left(q^{3}\right)[1-S(q)] \frac{\omega_{\mathrm{pl}}^{2}}{q^{2}}, \quad \omega_{\mathrm{pl}}^{2}=\frac{4 \alpha r_{s}}{3 \pi},
$$

respectively. In Equation (2.1), $\omega_{\mathrm{pl}}$ is the classical plasma frequency with $\alpha=[4 /(9 \pi)]^{1 / 3}$. The momenta $k, q$ are measured in units of $k_{\mathrm{F}}$, energies (including $\left.\omega_{\mathrm{pl}}\right)$ in units of $k_{\mathrm{F}}^{2}$ [in a.u. it is $\left.k_{\mathrm{F}}=1 /\left(\alpha r_{\mathrm{s}}\right)\right]$. The GGSB analysis [15] is based upon the decomposition $S(q)=S_{0}(q)+\Delta S(q)$ with the term

$$
S_{0}(q)=\left(\frac{3 q}{4}-\frac{q^{3}}{16}\right) \Theta(2-q)+\Theta(q-2), \quad \int_{0}^{\infty} \mathrm{d}\left(q^{3}\right)\left[1-S_{0}(q)\right]=1
$$


Table 1. The behavior of the cumulant SFs vs. $q$ with $\omega_{p 1}^{2}=q_{c}^{2} / 3=4 \alpha r_{s} /(3 \pi)$. Note that $C_{a}(q)=C_{d}(q)$ and $C_{p}(q)=C_{d}(q)-C_{x}(q)$. The index “ $a$ " means spin-antiparallel, " $p$ " means spin-parallel, $d$ means direct (2-line) diagram, and $x$ means the corresponding exchange (1-line) diagram. For the Löwdin parameter $c$ see Equation (4.1), the other correlation parameters are $1-g_{a}(0), c_{1}$, and $4 t-3 g_{p}^{\prime \prime}(0)$. In addition to the normalization integrals of the last column, it holds that $\int_{0}^{\infty} d q \cdot q^{4} C_{p}(q)=g_{p}^{\prime \prime}(0)-4 t / 3$, see Equation (4.12).

\begin{tabular}{|c|c|c|c|}
\hline CSF & $q \ll q_{c}$ & $q \rightarrow \infty$ & $\int_{0}^{\infty} d\left(q^{3}\right)$ \\
\hline$C_{d}(q)$ & $\frac{1}{2}\left(\frac{3 q}{4}-\frac{q^{3}}{16}-\frac{q^{2}}{2 \omega_{p 1}}\right)+O\left(\frac{q}{\omega_{p 1}}\right)^{4} \omega_{p 1}$ & $\left(\frac{g_{a}(0)}{q^{4}}+\frac{2 / 5+c_{1}}{q^{6}}+\cdots\right) \omega_{p 1}^{2}+\cdots$ & $1-g_{a}(0)$ \\
\hline$C_{x}(q)$ & $C_{\mathrm{xr}}(q)+\cdots$ & $\left(\frac{g_{a}(0)}{q^{4}}+\frac{3 g_{p}^{\prime \prime}(0)+2 / 5+c_{1}}{q^{6}}+\cdots\right) \omega_{p 1}^{2}+\cdots$ & $1-g_{a}(0)$ \\
\hline$C_{p}(q)$ & $\frac{1}{2}\left(\frac{3 q}{4}-\frac{q^{3}}{16}-\frac{q^{2}}{2 \omega_{p 1}}\right)-C_{x r}(q)+\cdots$ & $-\left(\frac{3 g_{p}^{\prime \prime}(0)}{q^{6}}+\cdots\right) \omega_{p 1}^{2}-$ & 0 \\
\hline
\end{tabular}

Table 2. The behavior of the structure factors (SFs) vs. the momentum transfer $q$. Note that $S_{a}(q)=-C_{a}(q)$ and $S_{p}(q)$ $=1-F(q)-C_{p}(q)$. The spin-summed SF is $S(q)=S_{p}(q)+S_{a}(q)=1-F(q)-C(q)$, the magnetic SF is $M(q)=$ $S_{p}(q)-S_{a}(q)$. In addition to the normalization integrals of the last column, it holds that $\int_{0}^{\infty} d q \cdot q^{4}\left[1-S_{p}(q)\right]=g_{p}^{\prime \prime}(0)$, see Equation (4.12). Comment on $S_{p}(q)$ : the small-q behavior of $C_{x}(q)-\Delta F(q)$ is not known so far, the assumption $C_{x r}(q)-\Delta F_{r}(q)=O(q)^{4}$ agrees with the Iwamoto expression for the total SF $S_{p}(q \rightarrow 0)$; the normalization of $S_{p}(q)$ is due to the Pauli principle.

\begin{tabular}{|c|c|c|}
\hline $\mathrm{SF}$ & $q \ll q_{c}$ & normalization \\
\hline$F(q)$ & $1-c-z_{F}^{2}\left(\frac{3 q}{4}-\frac{q^{3}}{16}\right)+\cdots$ & $\frac{g_{a}(0)}{4} \frac{\omega_{p 1}^{4}}{q^{8}}+\cdots$ \\
\hline$S_{a}(q)$ & $-\frac{1}{2}\left(\frac{3 q}{4}-\frac{q^{3}}{16}-\frac{q^{2}}{2 \omega_{p 1}}\right)+O\left(\frac{q}{\omega_{p 1}}\right)^{4} \omega_{p 1}$ & $-\left(\frac{g_{a}(0)}{q^{4}}+\frac{2 / 5+c_{1}}{q^{6}}+\cdots\right) \omega_{p 1}^{2}+\cdots$ \\
\hline$S_{p}(q)$ & $\frac{1}{2}\left(\frac{3 q}{4}-\frac{q^{3}}{16}+\frac{q^{2}}{2 \omega_{p 1}}\right)+\left[C_{\mathrm{xr}}(q)-\Delta F_{\mathrm{r}}(q)\right]+\cdots$ & $1+\left(\frac{3 g_{p}^{\prime \prime}(0)}{q^{6}}+\cdots\right) \omega_{p 1}^{2}+\cdots$ \\
\hline$S(q)$ & $\frac{q^{2}}{2 \omega_{p 1}}+O\left(\frac{q}{\omega_{p 1}}\right)^{4} \omega_{p 1}$ & $1-\left(\frac{g_{a}(0)}{q^{4}}-\frac{3 g_{p}^{\prime \prime}(0)-2 / 5-c_{1}}{q^{6}}+\cdots\right) \omega_{p 1}^{2}+\cdots \quad \int_{0}^{\infty} d\left(q^{3}\right)[1-S(q)]=2-g_{a}(0)$ \\
\hline$M(q)$ & $\left(\frac{3 q}{4}-\frac{q^{3}}{16}\right)+O\left(\frac{q}{\omega_{p 1}}\right)^{4} \omega_{p 1}$ & $1+\left(\frac{g_{a}(0)}{q^{4}}+\frac{3 g_{p}^{\prime \prime}(0)+2 / 5+c_{1}}{q^{6}}+\cdots\right) \omega_{p 1}^{2}+\cdots$ \\
\hline
\end{tabular}

of the ideal Fermi gas with $n_{0}(k)=\Theta(1-k)$ and the term $\Delta S(q)$ vanishing at the high-density limit $r_{s} \rightarrow 0$, which corresponds to "no Coulomb repulsion". The linear-cubic combination $3 q / 4-q^{3} / 16$ that appears several times in the following derivations may be regarded as the ideal-Fermi-gas term. $S_{0}(q)$ has a simple geometrical meaning: it is the average volume accessible to the occupied momentum when excited by a transfer momentum of amplitude $q$ [33]. The interaction energy in the lowest order [i.e. with $S(q) \approx S_{0}(q)$ ] equals $v_{\mathrm{F}} \approx v_{1}=-(3 / 4)^{2} \omega_{\mathrm{pl}}^{2}$.

It is interesting to note that the plasmon sum rule [34] requires $S(q)$ to possess the small-q asymptotics of 


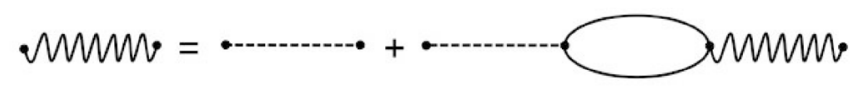

Figure 1. The Feynman diagrams for the Coulomb repulsion in RPA with the partial summation (or screening replacement) $v(q) \rightarrow v(q, \eta)$, the dashed line denotes the bare interaction $v(q)=q_{\mathrm{c}}^{2} / q^{2}$, the closed loop denotes the particle-hole propagator $Q(q, \eta)$ of Equation (B.3), and the wavy line denotes the effectively screened interaction $v(q, \eta)=v(q) /[1+v(q) Q(q, \eta)]$, where $q$ is the momentum transfer, $\eta$ is the energy or frequency.

$S(q)=q^{2} /\left(2 \omega_{\mathrm{pl}}\right)+\cdots$, where $\omega_{\mathrm{pl}}$ is the classical plasma frequency (in units of $\left.k_{\mathrm{F}}^{2}\right)$. [From the $f$-sum rule or Thomas-Reich-Kuhn sum rule of the dynamic SF $S(q, \omega)$ follows the plasmon sum rule $S\left(q \ll q_{c}\right)=q^{2} /\left(2 \omega_{\mathrm{pl}}\right)+\cdots$ of the static SF $S(q)$.] At first glance, this asymptotic behavior appears incompatible with the linear dependence of $S_{0}(q)$ on $q$ around $q=0$. However, these two observations are readily reconciled by writing

$$
S(q)=\frac{S_{0}(q)}{1+\Phi\left(\frac{3 \omega_{\mathrm{pl}}}{2 q}\right)},
$$

where $\Phi(x)$ is a function with the asymptotics $\Phi(x \rightarrow \infty) \rightarrow x$ and $\Phi(x \rightarrow 0) \rightarrow 0$.

In this paper, an alternative is presented, namely

$$
S(q)=1-F(q)-C(q) \text { with } F(q)=\int \frac{\mathrm{d}^{3} k}{4 \pi / 3} n(k) n(|\boldsymbol{k}+\boldsymbol{q}|), \quad \int_{0}^{\infty} \mathrm{d}\left(q^{3}\right) F(q)=1 .
$$

Note that what is called $\frac{1}{2} F(q)$ in [10] is named here $F(q)$. Equation (2.4) is the so-called cumulant decomposition into the "Hartree-Fock" component $1-F(q)$, reducible to the 1-body quantity $n(k)$, which follows from the exact 1-matrix, and the non-reducible "cumulant" component $C(q)$, such that for $r_{s} \rightarrow 0$ it is $C(q) \rightarrow 0$ and consequently $1-F(q) \rightarrow S_{0}(q)$. In this cumulant decomposition, the Coulomb repulsion $r_{s} / q^{2}$ enters the SF $S(q)$ at two places: 1) the "Fock term" $F(q)$ via the correlated momentum distribution $n(k)$ and 2) the cumulant SF $C(q)$ via linked diagrams, whereas in the GGSB decomposition the Coulomb repulsion is hidden only in $\Delta S(q)=S(q)-S_{0}(q)$ that (in the cumulant "language") equals $-\Delta F(q)-C(q)$ with $\Delta F(q)=F(q)-F_{0}(q)$ [which results from $\Delta n(k)=n(k)-n_{0}(k)$ ]. For plots of $F(q)$ see Figure 1 in paper I of [35]. The Fock term $F(q)$ brings in the momentum distribution $n(k)$ with its nonanalytical peculiarities, caused by the RPA long-range correlation. The complexity of the HEG manifests itself in nonanalyticities or singularities of the type $r_{s} \ln r_{s}$ or $q \ln q$, see e.g. $n(k \rightarrow 1)$ or $F(q \rightarrow 0)$. This gives rise to the $k$ - or $q$-dependences in relation to the dependence on $r_{s}$. For example, according to the perturbation theory, $n(k)$ should behave like $r_{s}^{2}$ for small $r_{s}$. This is true for $k$ values away from the Fermi edge (i.e. $k \gg 1$ and $k \ll 1$ ). However, at the Fermi edge $n(k)$ involves terms like $\sqrt{r_{s}}|k-1| \ln |k-1|$ or it jumps as $1-z_{\mathrm{F}} \sim r_{s}$. This implies a contribution $\Delta t \sim \int \mathrm{d}\left(k^{3}\right) k^{2} \Delta n(k)$ that equals $\sim r_{s}^{2} \ln r_{s}$, see [29].

The cumulant decomposition seems to be more complicated than its conventional counterpart but, on the other hand, it may be considered a more natural partitioning. This is so because, within the many-body perturbation theory, the cumulant SF $C(q)$ is given by linked diagrams, whereas $\Delta S(q)$ contains also unlinked diagrams.

As already mentioned above, the spin-summed SF is given by $S(q)=S_{\mathrm{a}}(q)+S_{\mathrm{p}}(q)$. In terms of the cumulant SFs $C_{\mathrm{a}, \mathrm{p}}(q)$, these quantities read

$$
S_{\mathrm{a}}(q)=-C_{\mathrm{a}}(q), \quad S_{\mathrm{p}}(q)=1-F(q)-C_{\mathrm{p}}(q),
$$

where $C(q)=C_{\mathrm{a}}(q)+C_{\mathrm{p}}(q)$, in agreement with Equation (2.4). The negative sign in front of $C_{\mathrm{a}, \mathrm{p}}(q)$ ensures the positive normalization of the cumulant SFs. Note that $S_{\mathrm{a}, \mathrm{p}}(0)=0$ (the perfect screening sum rule) implies $C_{\mathrm{a}}(0)=0$ and $C_{\mathrm{p}}(0)=C_{\mathrm{x}}(0)=1-F(0)$, the latter relating the 1-body quantity $n(k)$ to the 2-body 
quantities $C_{\mathrm{p}, \mathrm{x}}(q)$. The analogs of Equations (2.4) and (2.5) in the direct space, including Fourier transforms and normalizations, are given in Appendix A together with the cusp and curvature theorems for $g_{\mathrm{a}}(0)$ and $g_{\mathrm{p}}^{\prime \prime}(0)$, respectively, which are short-range correlation parameters of $g_{\mathrm{a}, \mathrm{p}}(r)$.

The original definitions of the 1- and 2-body RDMs in terms of the anticommuting creation and annihiliation operators $\psi^{\dagger}(\boldsymbol{r}, \sigma)$ and $\psi(\boldsymbol{r}, \sigma)$, respectively, are given by

$$
\gamma_{1}\left(1 \mid 1^{\prime}\right)=\left\langle\Phi_{0}\left|\psi_{1^{\prime}}^{\dagger} \psi_{1}\right| \Phi_{0}\right\rangle, \quad \gamma_{2}\left(1\left|1^{\prime}, 2\right| 2^{\prime}\right)=\left\langle\Phi_{0}\left|\psi_{1^{\prime}}^{\dagger} \psi_{2^{\prime}}^{\dagger} \psi_{2} \psi_{1}\right| \Phi_{0}\right\rangle .
$$

The short hands $1=\left(\boldsymbol{r}_{1}, \sigma_{1}\right)$ and $\int \mathrm{d} 1=\sum_{\sigma_{1}} \int \mathrm{d}^{3} r_{1}$ are used throughout the text, $1^{\prime}=\left(\boldsymbol{r}_{1}^{\prime}, \sigma_{1}^{\prime}\right)$ [instead of $\left.\left(\boldsymbol{r}_{1^{\prime}}, \sigma_{1^{\prime}}\right)\right]$. The hermiticity of $\gamma_{2}$ is obvious and the permutational antisymmetry means that $\gamma_{2} \rightarrow-\gamma_{2}$ when 1 and 2 or $1^{\prime}$ and $2^{\prime}$ are interchanged. $\hat{N}=\int \mathrm{d} 1 \psi_{1}^{\dagger} \psi_{1}$ is the total particle-number operator. The ground state $\Phi_{0}$ is an $N$ body state, hence $\psi_{1}\left|\Phi_{0}\right\rangle$ and $\left\langle\Phi_{0}\right| \psi_{1}^{\dagger}$ are $(N-1)$-body states, and thus

$$
\int \mathrm{d} 1\left\langle\Phi_{0}\left|\psi_{1}^{\dagger} \psi_{1}\right| \Phi_{0}\right\rangle=N, \quad \int \mathrm{d} 1 \mathrm{~d} 2\left\langle\Phi_{0}\left|\psi_{1}^{\dagger} \psi_{2}^{\dagger} \psi_{2} \psi_{1}\right| \Phi_{0}\right\rangle=N(N-1)
$$

holds for the normalization. The corresponding contraction sum rule

$$
\int \mathrm{d} 2\left\langle\Phi_{0}\left|\psi_{1}^{\dagger} \psi_{2}^{\dagger} \psi_{2} \psi_{1}\right| \Phi_{0}\right\rangle=\left\langle\Phi_{0}\left|\psi_{1^{\prime}}^{\dagger} \hat{N} \psi_{1}\right| \Phi_{0}\right\rangle=\gamma_{1}\left(1 \mid 1^{\prime}\right)(N-1)
$$

describes how the 1-matrix $\gamma_{1}$ for finite $N$ results from the 2-matrix $\gamma_{2}$. Equation (2.8) does not permit the above mentioned thermodynamic limit.

A way towards a size-extensive contraction is the cumulant decomposition. This means to define the cumulant 2-matrix $\gamma_{\mathrm{c}}\left(1\left|1^{\prime}, 2\right| 2^{\prime}\right)$ with

$$
\gamma_{2}\left(1\left|1^{\prime}, 2\right| 2^{\prime}\right)=\gamma_{1}\left(1 \mid 1^{\prime}\right) \gamma_{1}\left(2 \mid 2^{\prime}\right)-\gamma_{1}\left(1 \mid 2^{\prime}\right) \gamma_{1}\left(2 \mid 1^{\prime}\right)-\gamma_{c}\left(1\left|1^{\prime}, 2\right| 2^{\prime}\right) .
$$

This matrix obviously has the spin structure

$$
\gamma_{\mathrm{c}}\left(1\left|1^{\prime}, 2\right| 2^{\prime}\right)=\delta_{-}\left(\sigma_{1}\left|\sigma_{1}^{\prime}, \sigma_{2}\right| \sigma_{2}^{\prime}\right) \gamma_{\mathrm{c}}^{+}\left(\boldsymbol{r}_{1}\left|\boldsymbol{r}_{1}^{\prime}, \boldsymbol{r}_{2}\right| \boldsymbol{r}_{2}^{\prime}\right)+\delta_{+}\left(\sigma_{1}\left|\sigma_{1}^{\prime}, \sigma_{2}\right| \sigma_{2}^{\prime}\right) \gamma_{\mathrm{c}}^{-}\left(\boldsymbol{r}_{1}\left|\boldsymbol{r}_{1}^{\prime}, \boldsymbol{r}_{2}\right| \boldsymbol{r}_{2}^{\prime}\right)
$$

with the symmetric and antisymmetric 2-body spin functions

$$
\delta_{ \pm}\left(\sigma_{1}\left|\sigma_{1}^{\prime}, \sigma_{2}\right| \sigma_{2}^{\prime}\right)=\frac{1}{2}\left(\delta_{\sigma_{1}, \sigma_{1}^{\prime}} \delta_{\sigma_{2}, \sigma_{2}^{\prime}} \pm \delta_{\sigma_{1}, \sigma_{2}^{\prime}} \delta_{\sigma_{2}, \sigma_{1}^{\prime}}\right) \text {, where } \frac{1}{4} \sum_{\sigma_{1,2}} \delta_{ \pm}\left(\sigma_{1}\left|\sigma_{1}, \sigma_{2}\right| \sigma_{2}\right)=\left\{\begin{array}{l}
3 / 4 \\
1 / 4
\end{array}\right. \text {. }
$$

Equations (2.10) and (2.11) define $\gamma_{c}^{+}$(which does not change sign under the exchange $\boldsymbol{r}_{1} \leftrightarrow \boldsymbol{r}_{2}$ or $\boldsymbol{r}_{1}^{\prime} \leftrightarrow \boldsymbol{r}_{2}^{\prime}$ ) and $\gamma_{c}^{-}$(which changes sign under these exchanges). With $\gamma_{c}^{ \pm}=\left(\rho^{2} / 4\right) \chi_{ \pm}$, the dimensionless cumulant 2-matrices $\chi_{ \pm}$are introduced. The next step involves deriving $\chi_{\mathrm{a}}$ (for electron pairs with antiparallel spins) and $\chi_{\mathrm{p}}$ (for electron pairs with parallel spins) from $\chi_{ \pm}$. With $\sigma_{1}^{\prime}=\sigma_{1}$ and $\sigma_{2}^{\prime}=\sigma_{2}$ together with $\sigma_{1}=-\sigma_{2}$ for "a" or $\sigma_{1}=\sigma_{2}$ for "p", Equation (2.10) leads to

$$
\chi_{\mathrm{a}}=\frac{1}{2}\left(\chi_{+}+\chi_{-}\right) \text {and } \chi_{\mathrm{p}}=\chi_{-} \text {, }
$$

respectively. Here and in the following, the arguments $\left(\boldsymbol{r}_{1}\left|\boldsymbol{r}_{1}^{\prime}, \boldsymbol{r}_{2}\right| \boldsymbol{r}_{2}^{\prime}\right)$ of these matrices are suppressed for the sake of convenience. Their diagonals (for which $\boldsymbol{r}_{1}^{\prime}=\boldsymbol{r}_{1}$ and $\boldsymbol{r}_{2}^{\prime}=\boldsymbol{r}_{2}$ ) are denoted by $h_{ \pm}\left(r_{12}\right)$ and $h_{\mathrm{a}, \mathrm{p}}\left(r_{12}\right)$. They determine the normalizations

$$
\alpha^{3} \int_{0}^{\infty} \mathrm{d}\left(r^{3}\right) \frac{1}{2} h_{ \pm}(r)=\mp c \Rightarrow \alpha^{3} \int_{0}^{\infty} \mathrm{d}\left(r^{3}\right) \frac{1}{2} h_{\mathrm{a}}(r)=0, \quad \alpha^{3} \int_{0}^{\infty} \mathrm{d}\left(r^{3}\right) \frac{1}{2} h_{\mathrm{p}}(r)=c,
$$

where $c=1-F(0) \geq 0$. Their Fourier transforms (with the integral operator $\hat{T}$ ) yield the desired cumulant SFs,

$$
\hat{T} \phi(r)=\alpha^{3} \int_{0}^{\infty} \mathrm{d}\left(r^{3}\right) \frac{\sin q r}{q r} \phi(r), \quad C_{ \pm}(q)=\hat{T} \frac{1}{2} h_{ \pm}(r), \quad C_{\mathrm{a}, \mathrm{p}}(q)=\hat{T} \frac{1}{2} h_{\mathrm{a}, \mathrm{p}}(r),
$$

see also Appendix A. A consequence of Equation (2.14) and Equations (A.6)-(A.8) is that the short-range correlation determines the asymptotic large- $q$ behavior of the SFs and their normalizations.

Where do the cumulant 2-matrices $\chi_{ \pm}$come from? As mentioned above, they are given by linked diagrams. 
Each direct diagram $\chi_{\mathrm{d}_{1}}\left(\boldsymbol{r}_{1}\left|\boldsymbol{r}_{1}^{\prime}, \boldsymbol{r}_{2}\right| \boldsymbol{r}_{2}^{\prime}\right)$ with two open particle-hole lines (one running from $\boldsymbol{r}_{1}^{\prime}$ to $\boldsymbol{r}_{1}$ and another one from $\boldsymbol{r}_{2}^{\prime}$ to $\left.\boldsymbol{r}_{2}\right)$ is associated with an exchange diagram $\chi_{\mathrm{x}_{1}}\left(\boldsymbol{r}_{1}\left|\boldsymbol{r}_{1}^{\prime}, \boldsymbol{r}_{2}\right| \boldsymbol{r}_{2}^{\prime}\right)=\chi_{\mathrm{d}_{1}}\left(\boldsymbol{r}_{1}\left|\boldsymbol{r}_{2}^{\prime}, \boldsymbol{r}_{2}\right| \boldsymbol{r}_{1}^{\prime}\right)$, see for example Figure 2(a) and Figure 3(a). Thus, a series of diagram pairs $\left(\mathrm{d}_{1}, \mathrm{x}_{1}\right),\left(\mathrm{d}_{2}, \mathrm{x}_{2}\right), \cdots$ exists from which the building elements $\chi_{\mathrm{d}}=\chi_{\mathrm{d}_{1}}+\chi_{\mathrm{d}_{2}}+\cdots$ and $\chi_{\mathrm{x}}=\chi_{\mathrm{x}_{1}}+\chi_{\mathrm{x}_{2}}+\cdots$ follow. The next step defines the singlet/triplet components $\chi_{ \pm}=\chi_{\mathrm{d}} \pm \chi_{\mathrm{x}}$ in terms of these diagrams, the relations

$$
\left.\begin{array}{l}
\chi_{\mathrm{a}}=\chi_{\mathrm{d}} \\
\chi_{\mathrm{p}}=\chi_{\mathrm{d}}-\chi_{\mathrm{x}}
\end{array}\right\} \Rightarrow \chi=\frac{1}{2}\left(\chi_{\mathrm{a}}+\chi_{\mathrm{p}}\right)=\frac{1}{4} \chi_{+}+\frac{3}{4} \chi_{-}=\chi_{\mathrm{d}}-\frac{1}{2} \chi_{\mathrm{x}}
$$

following from Equation (2.12). So, the spin-averaged sum $\chi$ can be written differently: The a- and p-components $\chi_{\mathrm{a}, \mathrm{p}}$ are equally weighted, whereas the singlet- and triplet-components $\chi_{ \pm}$have the weights equaling $1 / 4$ and $3 / 4$, and the components $\chi_{\mathrm{d}, \mathrm{x}}$ have the weights equaling 1 and $-1 / 2$, respectively. The same holds for the PDs $h_{\mathrm{a}, \mathrm{p}}(r)$. On the other hand, for the cumulant SFs $C_{\mathrm{a}, \mathrm{p}}(q)$ one has:

$$
\left.\begin{array}{l}
C_{\mathrm{a}}(q)=C_{\mathrm{d}}(q) \\
C_{\mathrm{p}}(q)=C_{\mathrm{d}}(q)-C_{\mathrm{x}}(q)
\end{array}\right\} \Rightarrow C(q)=C_{\mathrm{a}}(q)+C_{\mathrm{p}}(q)=2 C_{\mathrm{d}}(q)-C_{\mathrm{x}}(q) .
$$

Note the slightly different definitions of the spin-summed quantities $\chi, h$, and $g$ on one hand, and $C$ and $S$ on the other.

With $\chi_{\mathrm{d}}$ and $\chi_{\mathrm{x}}$ given from the Feynman diagrams and with $C_{\mathrm{d}}(q)$ and $C_{\mathrm{x}}(q)$ following from them, the spin-resolved components of the interaction energy $v=v_{\mathrm{a}}+v_{\mathrm{p}}$ are

$$
v_{\mathrm{a}}=-\frac{3 \omega_{\mathrm{pl}}^{2}}{4} \int_{0}^{\infty} \mathrm{d} q C_{\mathrm{a}}(q), \quad C_{\mathrm{a}}(q)=C_{\mathrm{d}}(q),
$$

and

$$
v_{\mathrm{p}}=-\frac{3 \omega_{\mathrm{p}}^{2}}{4} \int_{0}^{\infty} \mathrm{d} q\left[F(q)+C_{\mathrm{p}}(q)\right], \quad C_{\mathrm{p}}(q)=C_{\mathrm{d}}(q)-C_{\mathrm{x}}(q),
$$

which can be written as $v_{\mathrm{p}}=v_{\mathrm{F}}+v_{\mathrm{d}}+v_{\mathrm{x}}$. Thus, $v=v_{\mathrm{a}}+v_{\mathrm{p}}=v_{\mathrm{F}}+2 v_{\mathrm{d}}+v_{\mathrm{x}}, v_{\mathrm{F}}=-(3 / 4)^{2} \omega_{\mathrm{pl}}^{2}+\cdots$. In the following, RPA is used (index " $r$ ") and the terms $\Delta C_{\mathrm{d}}$ and $\Delta C_{\mathrm{x}}$ mean all the (hopefully small) contributions beyond RPA: $C_{\mathrm{d}}=C_{\mathrm{dr}}+\Delta C_{\mathrm{d}}$ and $C_{\mathrm{x}}=C_{\mathrm{xr}}+\Delta C_{\mathrm{x}}$.

\section{Antiparallel Spins: The Coulomb Hole}

We move through the chain $\chi_{\mathrm{d}} \rightarrow h_{\mathrm{d}} \rightarrow C_{\mathrm{d}} \rightarrow C_{\mathrm{a}} \rightarrow S_{\mathrm{a}}$, where "a" means antiparallel spins and "d" means "direct" diagrams (of the type depicted in Figure 2(a)). In its Cartesian space representation, $\chi_{\mathrm{dr}}$ is given by (using the definitions of Appendix B)

$$
\chi_{\mathrm{dr}}\left(\boldsymbol{r}_{1}\left|\boldsymbol{r}_{1}^{\prime}, \boldsymbol{r}_{2}\right| \boldsymbol{r}_{2}^{\prime}\right)=\frac{9}{2} \int \frac{\mathrm{d}^{3} q}{4 \pi} \frac{1}{2}\left\{\int \frac{\mathrm{d} \eta}{2 \pi \mathrm{i}} v(q, \eta) \hat{Q}_{1}(q, \eta) \hat{Q}_{2}(q,-\eta) \mathrm{e}^{\mathrm{i}\left(\boldsymbol{k}_{1} \boldsymbol{r}_{11^{\prime}}-\boldsymbol{k}_{2} \boldsymbol{r}_{22^{\prime}}+\boldsymbol{q}_{\mathbf{r}_{2} 2}\right)}+\text { h.c. }\right\}
$$

with the abbreviations $\boldsymbol{r}_{12}=\boldsymbol{r}_{1}-\boldsymbol{r}_{2}$ and $\boldsymbol{r}_{11^{\prime}}=\boldsymbol{r}_{1}-\boldsymbol{r}_{1}^{\prime}$. [Remember to read $\boldsymbol{r}_{1}^{\prime}$ instead of $\boldsymbol{r}_{1^{\prime}}$.] The integral operator $\hat{Q}_{i}(q, \eta)$ generalizes the particle-hole propagator $Q(q, \eta)$, see Equation (B.4). The diagonal elements $\chi_{\mathrm{dr}}\left(\boldsymbol{r}_{1}\left|\boldsymbol{r}_{1}, \boldsymbol{r}_{2}\right| \boldsymbol{r}_{2}\right)=h_{\mathrm{dr}}\left(r_{12}\right)$ define the cumulant PD [see Equation (B.5)]

$$
h_{\mathrm{dr}}(r)=\frac{9}{2} \int \frac{\mathrm{d}^{3} q}{4 \pi} q \frac{\sin q r}{q r} \int_{0}^{\infty} \frac{\mathrm{d} u}{\pi} R^{2}(q, u) \frac{q_{c}^{2}}{q^{2}+q_{c}^{2} R(q, u)} .
$$

When going from Equation (3.1) to Equation (3.2), the contour integration $\eta \rightarrow \mathrm{i} q u$, which replaces the frequency integration by the velocity integration, leads to the real function $R(q, u)=Q(q, \mathrm{i} q u)$ for the particlehole propagator, see e.g. Equation (B.1) in [11]. This leads to Equation (B.5) taking the form $I(q)$ $=8 \pi q \int_{0}^{\infty} \mathrm{d} u R^{2}(q, u)$, where $I(q)$ is referred to as the Macke function [23] [36] [37]. The effective interaction $v(q, \mathrm{i} q u)=v(q) /[1+v(q) R(q, u)]=q_{\mathrm{c}}^{2} /\left[q^{2}+q_{\mathrm{c}}^{2} R(q, u)\right]=w(q, u)$ is the RPA screened Coulomb interaction with a Yukawa-like cut-off $q_{\mathrm{c}}^{2} R(q, u), \quad q_{\mathrm{c}}^{2}=3 \omega_{\mathrm{pl}}^{2}=4 \alpha r_{\mathrm{s}} / \pi$. The reverse replacement $w(q, u) \rightarrow v(q)$ cancels out with $h_{\mathrm{dr}}(r) \rightarrow h_{\mathrm{d} 1}(r)$, thus restoring the original perturbation expression in the lowest order. The 


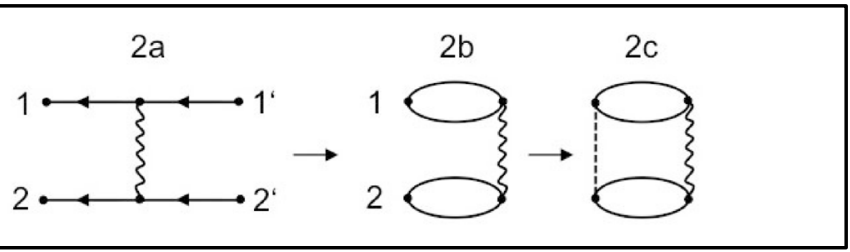

Figure 2. 2a denotes the lowest-order renormalized cumulant 2-matrix $\chi_{\mathrm{dr}}$, 2b denotes the non-divergent cumulant PD $h_{\mathrm{dr}}$ or the cumulant SF $C_{\mathrm{dr} r}$ (Yasuhara) [46], (Kimball) [38], and 2c denotes the non-divergent interaction energy $v_{\mathrm{d} r}$ (Macke, Gell-Mann/Brueckner) [23] [24].

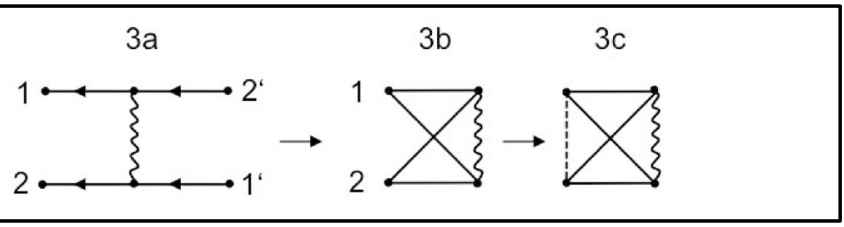

Figure 3. The RPA exchange terms corresponding to Figure 2 with 3a corresponding to $\chi_{\mathrm{xr}}$ (following from 2a corresponding to $\chi_{\mathrm{dr}}$ through the exchange replacement $1^{\prime} \leftrightarrow 2^{\prime}$, $3 \mathbf{b}$ corresponding to $h_{\mathrm{xr}}$ or $C_{\mathrm{xr}}$ (nondivergent), and 3c corresponding to $v_{\mathrm{xr}}$. As shown by Onsager et al. [54], already $v_{\mathrm{x} 2}$ does not diverge (needs no RPA renormalization). For the importance of " $x$ " see [40].

qualitative behavior of $h_{\mathrm{a}}(r)=h_{\mathrm{d}}(r)$ is depicted in Figure 11 of [7]; it begins at $r=0$ as a positive number and then decreases, becoming negative, and finally approaches zero from below, ensuring that its normalization vanishes, see Equation (A.6).

The Fourier transform of $h_{\mathrm{dr}}(r)$ is the cumulant SF $C_{\mathrm{d}}(q)$ within RPA:

$$
C_{\mathrm{dr}}(q)=\int \frac{\mathrm{d}^{3} r}{3 \pi^{2}} \cos \boldsymbol{q} r \frac{1}{2} h_{\mathrm{dr}}(r)=\frac{\omega_{\mathrm{pl}}^{2}}{(4 \pi / 3)^{2}} \frac{I_{\mathrm{dr}}(q)}{q^{2}}, \quad I_{\mathrm{dr}}(q)=8 \pi q \int_{0}^{\infty} \mathrm{d} u \frac{R^{2}(q, u)}{1+v(q) R(q, u)}
$$

[38]. Note that $I_{\mathrm{dr}}(q)$ parametrically depends on $r_{s}$, approaching the Macke function $I(q)$ for $r_{s} \rightarrow 0$ [with the properties (B.8), see also Figure 4]; $I_{\mathrm{d} r}(q)$ may be referred to as the "generalized Macke function". $C_{\mathrm{dr}}(q)$ determines $S_{\mathrm{a}}(q)$ via $C_{\mathrm{d}}=C_{\mathrm{a}}=-S_{\mathrm{a}}$, which yields the interaction energy of the antiparallel spin pairs

$$
v_{\mathrm{a}}=\frac{3 \omega_{\mathrm{pl}}^{2}}{4} \int_{0}^{\infty} \mathrm{d} q S_{\mathrm{a}}(q)=-\frac{3}{4}\left(\frac{\omega_{\mathrm{pl}}^{2}}{4 \pi / 3}\right)^{2} \int_{0}^{\infty} \mathrm{d} q \frac{I_{\mathrm{dr}}(q)}{q^{2}}+\Delta v_{\mathrm{a}}=\left(\alpha r_{\mathrm{s}}\right)^{2}\left(a \ln r_{\mathrm{s}}-0.063\right)+\cdots
$$

with $a=2(1-\ln 2) / \pi^{2} \approx 0.062$ at the high-density limit. The short-range correlation parameter

$$
1-g_{\mathrm{a}}(0)=-\int_{0}^{\infty} \mathrm{d}\left(q^{3}\right) S_{\mathrm{a}}(q)=3 \frac{\omega_{\mathrm{pl}}^{2}}{(4 \pi / 3)^{2}} \int_{0}^{\infty} \mathrm{d} q I_{\mathrm{dr}}(q)-\Delta g_{\mathrm{a}}(0)
$$

also follows from $I_{\mathrm{dr}}(q)$ in RPA with

$$
1-g_{\mathrm{a}}(0)=\frac{2}{5 \pi}\left(\pi^{2}+6 \ln 2-3\right) \alpha r_{\mathrm{s}}+2\left(3-\frac{\pi^{2}}{4}\right)\left(\frac{3 \alpha}{2 \pi}\right) r_{s}^{2} \ln r_{\mathrm{s}}+O\left(r_{s}^{2}\right)
$$

at the high-density limit [38]-[40]. Actually, it is exactly this high-density behavior of $h(r)$ and $g(r)$ for $r \rightarrow 0$ that results from the ladder theory, which constitutes the best method to treat the short-range correlation [41]-[46] (for the recent developments see [27]). The correctness of Equations (3.3)-(3.6) confirms the starting point (3.1). 


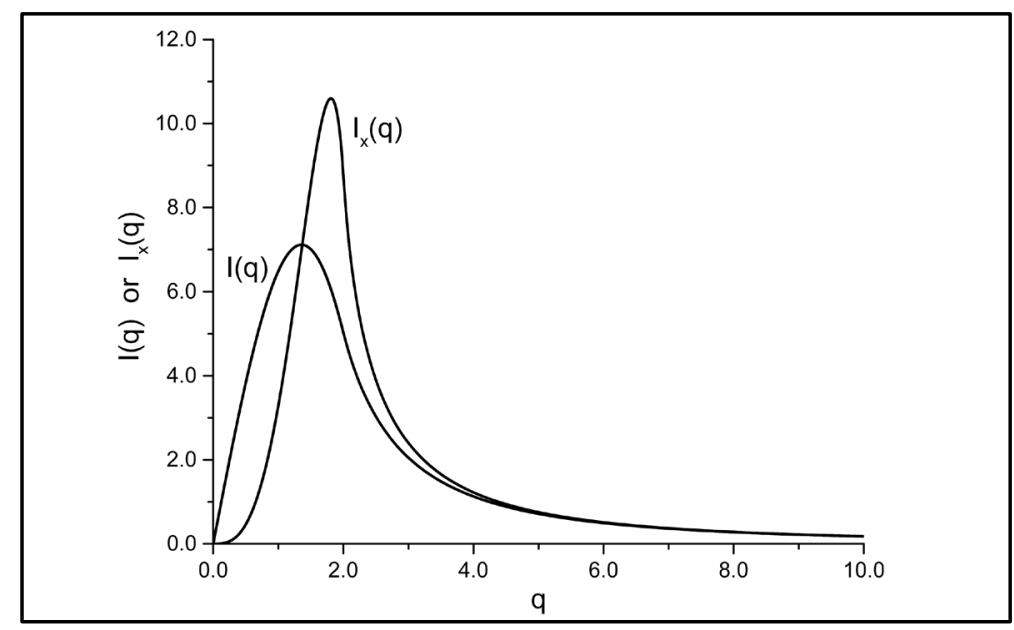

Figure 4. The functions $I(q)$ and $I_{x}(q)$.

How does $S_{\mathrm{a}}(q)=-C_{\mathrm{a}}(q)=-C_{\mathrm{d}}(q) \approx-C_{\mathrm{dr}}(q)$ behave for small $q$ ?

$$
S_{\mathrm{a}}\left(q \ll q_{c}\right)=-\frac{1}{2}\left(\frac{3 q}{4}-\frac{q^{3}}{16}-\frac{q^{2}}{2 \omega_{\mathrm{pl}}}\right)+\left[d_{4}\left(\frac{q}{\omega_{\mathrm{pl}}}\right)^{4}+d_{5}\left(\frac{q}{\omega_{\mathrm{pl}}}\right)^{5}+\cdots\right] \omega_{\mathrm{pl}}-\Delta C_{\mathrm{d}}(q)
$$

in agreement with an ansatz of Iwamoto [47]. The coefficients $d_{4,5}$ are due to multipair and quasiparticle-quasihole excitations, respectively. They follow from $I_{\mathrm{dr}}(q)$ as $d_{4}=-3 /\left(2^{3} \times 5\right)=-0.075$ and $d_{5}=23 /\left(2^{5} \times 5^{2}\right)=0.02875$ [48]. Note that the region $q \ll q_{c}$ shrinks with decreasing $r_{s}$ and finally vanishes for $r_{\mathrm{s}} \rightarrow 0$. As $S_{\mathrm{a}}(q)$ contains (in the paramagnetic gas) half of the plasmon term, the other half has to come from $S_{\mathrm{p}}(q)$, which also has to compensate the ( $r_{\mathrm{s}}$-independent) ideal-Fermi-gas term.

In the transitional region between the small and large values of $q$ there is a peculiar point. Namely, upon approaching from below the transition momenta of $|\boldsymbol{q}|=2$, the topology changes from two overlapping to two non-overlapping Fermi spheres. This topology change causes a jump discontinuity in $I^{\prime \prime}(q)$ and $S_{\mathrm{a}}^{\prime \prime}(q)$ at $q=2$ [36], namely

$$
\Delta I^{\prime \prime}(2)=2 \pi^{2} \Rightarrow \Delta S_{\mathrm{a}}^{\prime \prime}(2)=-\frac{1}{2}\left(\frac{3 \omega_{\mathrm{pl}}}{4}\right)^{2}+\cdots,
$$

the ellipsis representing the terms beyond RPA. In the Cartesian space these jump discontinuities cause the Friedel oscillations in $g_{\mathrm{a}}(r)$. They make interpolations between $q \rightarrow 0$ and $q \rightarrow \infty$ with Padé approximants [49] or the robust interpolation scheme [50] questionable.

How does $C_{\mathrm{dr}}(q)$ of Equation (3.3) behave for $q \rightarrow \infty$ ? As the large- $q$ asymptotics does not require the RPA, the perturbation theory holds in the lowest order, hence in Equation (3.3) the "descreening" replacement $w(q, u) \approx v(q)$ is possible. The integration over $u$ yields the Macke function $I(q)$ with the large- $q$ asymptotics (B.8) starting with $1 / q^{4}$. Thus, for $q \rightarrow \infty$ one has

$$
S_{\mathrm{a}}(q \rightarrow \infty)=\left[-\frac{1}{q^{4}}-\frac{2}{5} \frac{1}{q^{6}}+\cdots\right] \omega_{\mathrm{pl}}^{2}+\Delta S_{\mathrm{a}}(q \rightarrow \infty) .
$$

Are there perhaps corrections due to terms beyond RPA? Indeed, the electron-electron coalescence cusp theorem $g_{\mathrm{a}}^{\prime}(0)=\alpha r_{s} g_{\mathrm{a}}(0)$ together with the Kimball trick [51] makes the first term $\sim 1 / q^{4}$ decorated with an additional factor $g_{\mathrm{a}}(0) \leq 1$. One may expect the next term to be modified in a similar way, thus

$$
S_{\mathrm{a}}(q \rightarrow \infty)=\left[-g_{\mathrm{a}}(0) \frac{1}{q^{4}}-\left(\frac{2}{5}+c_{1}\right) \frac{1}{q^{6}}+\cdots\right] \omega_{\mathrm{pl}}^{2}+\tilde{\Delta} S_{\mathrm{a}}(q \rightarrow \infty)
$$

with unknown correlation parameters $1-g_{\mathrm{a}}(0)$ and $c_{1}$ vanishing for $r_{\mathrm{s}} \rightarrow 0$ and with the beyond-RPA term $\tilde{\Delta} S_{\mathrm{a}}(q)$ different from $\Delta S_{\mathrm{a}}(q)$. The meaning of Equation (3.10) is as follows: the short-range correlation 
parameter $1-g_{\mathrm{a}}(0)$ determines the large-wavenumber asymptotics of $S_{\mathrm{a}, \mathrm{p}}(q)$ [41] [51] [52]. Because $S_{\mathrm{a}}(q)$ depends on the parameter $g_{\mathrm{a}}(0)$, one may read Equation (3.5) as an integral equation to be solved iteratively. One may also ask how to modify the RPA-result $C_{\mathrm{dr}}(q)$ of Equation (3.3) in such a way that it absorbs the correct large- $q$ behavior (3.10).

\section{Parallel Spins: The Fermi Hole}

For the parallel-spin, $\mathrm{SF}$ it is appropriate to write it as $S_{\mathrm{p}}=S_{0}-\Delta F-C_{\mathrm{p}}$ with $S_{0}=1-F_{0}, \Delta F=F(q)-F_{0}$, and $C_{\mathrm{p}}=C_{\mathrm{d}}-C_{\mathrm{x}}$. Thus besides $C_{\mathrm{d}}(q)$ one also needs the quantities $\Delta F(q)$ and $C_{\mathrm{x}}(q)$, which arise from the same source, namely $\chi_{\mathrm{x}} \cdot \Delta F$ and $C_{\mathrm{p}}$ do not contribute to the normalization of $S_{\mathrm{p}}$, but they do contribute to the interaction energy $v$ [Equation (2.13)] and to the on-top Fermi hole curvature [Equation (4.12)]. Whereas $S_{0}$ describes the smooth transition from 0 for small $q$ to 1 for $q \geq 2$ as it is known from the idealFermi gas, the term $\Delta F+C_{d}-C_{x}$ describes the interaction induced corrections.

$\Delta F$ is known to some extent (at least sufficient for a qualitative discussion). As it can be seen from Figure 1 of [35], it is $\Delta F(q)<0$ for $q<q_{0}<2$ and $\Delta F>0$ for $q>q_{0}$ with $\Delta F\left(q_{0}\right)=0$ defining $q_{0}$. The Fock function $F(q)$ defined in Equation (2.4) contributes to $S_{\mathrm{p}}$. It has the properties (4.1)-(4.4):

$$
\Delta F(0)=-c \quad \text { with } c=\int_{0}^{\infty} \mathrm{d}\left(k^{3}\right) n(k)[1-n(k)] \text { or } 1-c=\int_{0}^{\infty} \mathrm{d}\left(k^{3}\right)[n(k)]^{2} .
$$

The quantity $c$ is referred to as the Löwdin parameter or the index of non-idempotency. [P.-O. Löwdin was first to address the meaning of the trace of the squared 1-matrix.] It is a correlation parameter that measures the correlation-induced non-idempotency of the momentum distribution $n(k)$ and vanishes for $r_{s} \rightarrow 0$. For the dependence of $c$ on $r_{s}$ see [7]. For the kinetic part of the correlation energy $\Delta t=t-t_{0}$ it holds

$$
\int_{0}^{\infty} \mathrm{d} q q^{4} \Delta F(q)=\frac{4}{3} \Delta t, \quad \Delta t=-\frac{a}{2}\left(\alpha r_{s}\right)^{2} \ln r_{s}+\cdots
$$

The small- and large- $q$ asymptotics of $\Delta F(q)$ are

$$
\Delta F(q \rightarrow 0)=-c+\left(1-z_{\mathrm{F}}^{2}\right)\left(\frac{3 q}{4}-\frac{q^{3}}{16}\right)+\cdots \quad \text { and } \quad \Delta F(q \rightarrow \infty)=\frac{g_{\mathrm{a}}(0)}{4} \frac{\omega_{\mathrm{pl}}^{4}}{q^{8}}+\cdots,
$$

respectively. The small $q$ behavior has been demonstrated with the decomposition $n(k)=z_{\mathrm{F}} \Theta(1-k)+n_{1}(k)$, where $n_{1}(k)$ is continuous (jump-free), see Equation (3.8) in [7] or Equation (5.1) in [35]. The quantity $z_{\mathrm{F}}=n\left(1^{-}\right)-n\left(1^{+}\right) \leq 1$ is the quasiparticle weight with $z_{\mathrm{F}} \approx 1-0.177 r_{s}+\cdots$ within RPA. Note the nonanalytical behavior of $\Delta F(q \rightarrow 0)$ with terms $\sim q^{2} \ln q+O\left(q^{2}\right)$ and note that here the prefactor of the ideal-Fermi-gas term depends on $r_{s}$. The Löwdin parameter $c$ and the term quadratic in $z_{\mathrm{F}}$ have to be canceled out by $C_{\mathrm{x}}(q)$ and replaced by the other half of the plasmon term. The large- $q$ behavior arises from $\left.n(k \rightarrow \infty)=g_{\mathrm{a}}(0) \omega_{\mathrm{pl}}^{4}\right)\left(4 k^{8}\right)+\cdots$ [52] [53], which is influenced by the short-range correlation parameter $g_{\mathrm{a}}(0)$, in analogy to $S_{\mathrm{a}, \mathrm{p}}(q \rightarrow \infty)$ in Equations (3.10) and (4.12), respectively. At $q \approx 2$, there is a singular (topologically caused) jump

$$
F(q \approx 2)=F(2)+F^{\prime}(2)(q-2)+z_{\mathrm{F}}^{2} \frac{3}{8}(q-2)^{2} \Theta(2-q)+\cdots \Rightarrow \Delta F^{\prime \prime}(2)=-\frac{3}{4} z_{\mathrm{F}}^{2}+\cdots .
$$

Together with $\Delta C_{\mathrm{p}}^{\prime \prime}(2)$ that arises from $\Delta I_{\mathrm{x}}^{\prime \prime}(2)$, it contributes to $\Delta S_{\mathrm{p}}^{\prime \prime}(2)=-\Delta F^{\prime \prime}(2)-\Delta C_{\mathrm{p}}^{\prime \prime}(2)$, the source of the Friedel oscillations in $g_{\mathrm{p}}(r)$, whereas Equation (3.8) leads to the Friedel oscillations of $g_{\mathrm{a}}(r)$.

Next, $C_{\mathrm{p}}(q)$ is to be considered. It follows from the Feynman diagrams that $\chi_{\mathrm{x}} \rightarrow h_{\mathrm{x}} \rightarrow C_{\mathrm{x}} \rightarrow C_{\mathrm{p}}$, where " $p$ " means parallel spins and " $x$ " stands for exchange. According to Figure $3(\mathrm{a})$, the exchange counterpart to $\chi_{\mathrm{dr}}$ is given by

$$
\chi_{\mathrm{xr}}\left(\boldsymbol{r}_{1}\left|\boldsymbol{r}_{1}^{\prime}, \boldsymbol{r}_{2}\right| \boldsymbol{r}_{2}^{\prime}\right)=\frac{9}{2} \int \frac{\mathrm{d}^{3} q}{4 \pi} \frac{1}{2}\left\{\int \frac{\mathrm{d} \eta}{2 \pi \mathrm{i}} \hat{Q}_{1}(q, \eta) \hat{Q}_{2}(q,-\eta) v(q, \eta) \mathrm{e}^{\mathrm{i}\left(\boldsymbol{k}_{1} \boldsymbol{r}_{12^{\prime}}-\boldsymbol{k}_{2} \boldsymbol{r}_{21^{\prime}}+\boldsymbol{q}_{r_{12}}\right)}+\text { h.c. }\right\} .
$$

Its diagonal elements define the cumulant PD (within RPA)

$$
h_{\mathrm{xr}}(r)=\left.\frac{9}{2} \int \frac{\mathrm{d}^{3} q}{4 \pi} \operatorname{Re} \int \frac{\mathrm{d} \eta}{2 \pi \mathrm{i}} \hat{Q}_{1}(q, \eta) \hat{Q}_{2}(q,-\eta) v(q, \eta) \frac{\sin k r}{k r}\right|_{k=k_{1}+\boldsymbol{k}_{2}+q},
$$


and (after a tedious derivation) its Fourier transform turns out to be equal

$$
\begin{aligned}
C_{\mathrm{xr}}(q) & =3 \int \frac{\mathrm{d}^{3} k_{1} \mathrm{~d}^{3} k_{2}}{(4 \pi)^{2}} \int_{-\infty}^{+\infty} \frac{k \mathrm{~d} u}{\pi} \frac{\varepsilon_{1} \varepsilon_{2}}{\left(k^{2} u^{2}+\varepsilon_{1}^{2}\right)\left(k^{2} u^{2}+\varepsilon_{2}^{2}\right)} \cdot \frac{q_{c}^{2}}{k^{2}+q_{c}^{2} R(k, u)}=\frac{\omega_{\mathrm{pl}}^{2}}{(4 \pi / 3)^{2}} \frac{I_{\mathrm{xr}}(q)}{q^{2}}, \\
k_{1,2} & <1,\left|\boldsymbol{k}_{1,2}+\boldsymbol{q}\right|>1, \boldsymbol{k}=\boldsymbol{k}_{1}+\boldsymbol{k}_{2}+\boldsymbol{q}, \varepsilon_{1}=t\left(\boldsymbol{k}_{2}+\boldsymbol{q}\right)-t\left(\boldsymbol{k}_{1}\right), \\
\varepsilon_{2} & =t\left(\boldsymbol{k}_{1}+\boldsymbol{q}\right)-t\left(\boldsymbol{k}_{2}\right), t(\boldsymbol{k})=\boldsymbol{k}^{2} / 2 .
\end{aligned}
$$

Unfortunately this complicated integral is not known so far as an explicit function of $q$ and $r_{\mathrm{s}}$. If it is approximately substituted by $C_{\mathrm{x} 1}(q)$, then the integration over $u$ yields the well-known energy denominator for the exchange term:

$$
\int_{-\infty}^{+\infty} \frac{\mathrm{d} x}{\pi} \frac{\varepsilon_{1} \varepsilon_{2}}{\left(x^{2}+\varepsilon_{1}^{2}\right)\left(x^{2}+\varepsilon_{2}^{2}\right)}=\frac{1}{\varepsilon_{1}+\varepsilon_{2}}=\frac{1}{\boldsymbol{q} \cdot\left(\boldsymbol{k}_{1}+\boldsymbol{k}_{2}+\boldsymbol{q}\right)},
$$

resulting in

$$
C_{\mathrm{x} 1}(q)=\frac{\omega_{\mathrm{pl}}^{2}}{(4 \pi / 3)^{2}} \frac{I_{\mathrm{x}}(q)}{q^{2}} \text { and } I_{\mathrm{x}}(q)=\int \frac{\mathrm{d}^{3} k_{1} \mathrm{~d}^{3} k_{2}}{\boldsymbol{q} \cdot\left(\boldsymbol{k}_{1}+\boldsymbol{k}_{2}+\boldsymbol{q}\right)} \frac{q^{2}}{\left(\boldsymbol{k}_{1}+\boldsymbol{k}_{2}+\boldsymbol{q}\right)^{2}}
$$

with $k_{1,2}<1,\left|\boldsymbol{k}_{1,2}+\boldsymbol{q}\right|>1$, in analogy to Equation (3.3). $I_{x}(q)$ is referred to as the Gutlé function [33]; for its definition and properties see Equations (B.9), (B.10), and Figure 4. The quantity $I_{\mathrm{xr}}(q)$ introduced above in Equation (4.7) can be regarded as its generalization. It is worth mentioning that, whereas in $C_{\mathrm{dr}}(q)$ the screened interaction $v(q, \eta)$ is necessary to remove the long-range divergences of the bare interaction $v(q)$, this is not the case for $v_{\mathrm{x} 2}$ as it follows from

$$
v_{\mathrm{x}}=\frac{3 \omega_{\mathrm{pl}}^{2}}{4} \int_{0}^{\infty} \mathrm{d} q C_{\mathrm{x}}(q) \Rightarrow v_{\mathrm{x} 2}=\frac{3}{4}\left(\frac{\omega_{\mathrm{pl}}^{2}}{4 \pi / 3}\right)^{2} \int_{0}^{\infty} \mathrm{d} q \frac{I_{\mathrm{x}}(q)}{q^{2}}=\left(\frac{3 \pi}{4}\right)^{2}\left[\frac{1}{3} \ln 2-\frac{3}{2} \frac{\zeta(3)}{\pi^{2}}\right] \omega_{\mathrm{pl}}^{4}=0.048\left(\alpha r_{\mathrm{s}}\right)^{2} .
$$

This is the famous result of Onsager, Mittag, and Stephen [54] [55]. The total interaction energy equals $v=v_{\mathrm{a}}+v_{\mathrm{p}}=v_{\mathrm{F}}+2 v_{\mathrm{d}}+v_{\mathrm{x}}$ with $v_{\mathrm{F}} \approx v_{1}, v_{\mathrm{d}} \approx v_{\mathrm{dr}}$, and $v_{\mathrm{x}} \approx v_{\mathrm{xr}} \approx v_{\mathrm{x} 2}$.

Within the approximation $C_{\mathrm{xr}}(q) \approx C_{\mathrm{x} 1}(q)$, the exchange analog of Equation (3.5) reads

$$
1-g_{\mathrm{a}}(0)=\int_{0}^{\infty} \mathrm{d}\left(q^{3}\right) C_{\mathrm{x}}(q)=\left[3 \frac{\omega_{\mathrm{pl}}^{2}}{(4 \pi / 3)^{2}} \int_{0}^{\infty} \mathrm{d} q I_{\mathrm{x}}(q)+\cdots\right]-\Delta g_{\mathrm{a}}(0),
$$

which agrees with Equation (3.5) in the corresponding approximation $I_{\mathrm{dr}}(q) \approx I(q)$ thanks to $\int_{0}^{\infty} \mathrm{d} q\left[I(q)-I_{\mathrm{x}}(q)\right]=0$, see Equations (B.8) and (B.9).

Furthermore, the on-top curvature $g_{\mathrm{p}}^{\prime \prime}(0)$ of the Fermi hole follows from $S_{\mathrm{p}}(q)$ and, vice versa, it determines the large- $q$ asymptotics of $S_{\mathrm{p}}(q)$,

$$
g_{\mathrm{p}}^{\prime \prime}(0)=\int_{0}^{\infty} \mathrm{d} q q^{4}\left[1-S_{\mathrm{p}}(q)\right], \quad S_{\mathrm{p}}(q \rightarrow \infty)=1+\frac{3 g_{\mathrm{p}}^{\prime \prime}(0)}{q^{6}}+\cdots .
$$

$F(q)$ does not contribute to the $1 / q^{6}$-term because its expansion begins with $1 / q^{8}$. The proof of Equation (4.12) employs the curvature theorem (A.11) and the Kimball trick. With $S_{p}=1-F-C_{p}$ and Equation (4.2) it is

$$
g_{\mathrm{p}}^{\prime \prime}(0)=\frac{4}{3} t+\int_{0}^{\infty} \mathrm{d} q q^{4} C_{\mathrm{p}}(q)=\left[\frac{4}{3} t_{0}+\frac{\omega_{\mathrm{pl}}^{2}}{(4 \pi / 3)^{2}} \int_{0}^{\infty} \mathrm{d} q q^{2}\left[I(q)-I_{\mathrm{x}}(q)\right]+\cdots\right]+\Delta g_{p}^{\prime \prime}(0) .
$$

Note, that $t-t_{0}$ begins at the second order with $t_{2}$ and $I(q)-I_{\mathrm{x}}(q) \sim 1 / q^{4}$, see Equation (B.8). The terms proportional to $1 / q^{2}$, which would cause the integral $\int \mathrm{d} q q^{2} \cdots$ to diverge, cancel each other. At the high-density limit one has [56] [57]

$$
g_{\mathrm{p}}^{\prime \prime}(0)=\frac{2}{5}-\frac{1}{35 \pi}\left(4 \pi^{2}-5+20 \ln 2\right) \alpha r_{s}+O\left(r_{s}^{2} \ln r_{s}\right)
$$


with $t_{0}=3 / 10$.

Next the small- $q$ behavior of $C_{\mathrm{xr}}(q)$ is studied. Since the integral that enters Equation (4.7) is not known in a closed form, understanding the small- $q$ behavior of $C_{\mathrm{p}}$ is not as straightforward as that of $C_{\mathrm{a}}$. For $C_{\mathrm{p}}=C_{\mathrm{d}}-C_{\mathrm{x}}$ with Equation (3.3) for $C_{\mathrm{d}}$ and Equation (4.7) for $C_{\mathrm{x}}$ it holds

$$
C_{\mathrm{p}}(q \rightarrow 0)=\left[\frac{1}{2}\left(\frac{3 q}{4}-\frac{q^{3}}{16}-\frac{q^{2}}{2 \omega_{\mathrm{pl}}}\right)+\cdots\right]-C_{\mathrm{xr}}(q)+\cdots,
$$

where the first ellipsis stands for higher-order terms [of the order $O(q)^{4}$ or $O(q)^{5}$ ] of Equation (3.3) and the second ellipsis means the beyond-RPA terms $\Delta C_{\mathrm{p}}=\Delta C_{\mathrm{d}}-\Delta C_{\mathrm{x}}$ beginning with $O(q)^{4}$. With $\Delta F$ introduced above, for $S_{\mathrm{p}}=\left(S_{0}-C_{\mathrm{d}}\right)+\left(C_{\mathrm{x}}-\Delta F\right)$ it follows (within RPA) that

$$
S_{\mathrm{p}}(q \rightarrow 0)=\left[\frac{1}{2}\left(\frac{3 q}{4}-\frac{q^{3}}{16}+\frac{q^{2}}{2 \omega_{\mathrm{pl}}}\right)+\cdots\right]+\left[C_{\mathrm{xr}}(q)-\Delta F_{\mathrm{r}}(q)+\cdots\right] .
$$

What contributes to the second term as a function of $q$ and $r_{s}$ remains to be studied. If we assume that its power expansion begins with $O(q)^{4}$ then, in analogy with Equation (3.7), one obtains

$$
S_{\mathrm{p}}(q \rightarrow 0)=\frac{1}{2}\left(\frac{3 q}{4}-\frac{q^{3}}{16}+\frac{q^{2}}{2 \omega_{\mathrm{pl}}}\right)+O(q)^{4}
$$

and thus (again for $q \rightarrow 0$ )

$$
S(q)=S_{\mathrm{p}}(q)+S_{\mathrm{a}}(q)=\frac{q^{2}}{2 \omega_{\mathrm{pl}}}+\cdots, \quad M(q)=S_{\mathrm{p}}(q)-S_{\mathrm{a}}(q)=\left(\frac{3 q}{4}-\frac{q^{3}}{16}\right)+\cdots,
$$

in agreement with the plasmon sum rule and [15]. Note that $C_{\mathrm{x}}(q)$ and $n(k)$ [from which $F(q)$ follows] are indirectly related due to the fact that they have their common origin in the same Feynman diagrams. Namely, the starting point is the 4-point function $\chi_{\mathrm{x}}\left(\boldsymbol{r}_{1}\left|\boldsymbol{r}_{1}^{\prime}, \boldsymbol{r}_{2}\right| \boldsymbol{r}_{2}^{\prime}\right)$ (see Figure 3(a) and also Figure 3(a) in [10]). Upon carrying out the contraction procedure $\boldsymbol{r}_{2}^{\prime}=\boldsymbol{r}_{2}$, followed by integration over $\boldsymbol{r}_{2}$ and the Fourier transform for $\boldsymbol{r}_{1}-\boldsymbol{r}_{1^{\prime}}$, the source quantity for the momentum distribution $n(k)$ emerges, see [10]. On the other hand, upon taking the diagonal elements $\boldsymbol{r}_{1}^{\prime}=\boldsymbol{r}_{1}$ and $\boldsymbol{r}_{2}^{\prime}=\boldsymbol{r}_{2}$ and carrying out the Fourier transform for $\boldsymbol{r}_{12}=\boldsymbol{r}_{1}-\boldsymbol{r}_{2}$ one obtains $C_{\mathrm{x}}(q)$. Certainly a further study of $\Delta F_{\mathrm{r}}(q)$ and $C_{\mathrm{xr}}(q)$ within RPA and the beyond-RPA terms is warranted.

Next, the large- $q$ behavior of $C_{\mathrm{xr}}(q)$ is studied. For $q \rightarrow \infty$, the RPA quantity $C_{\mathrm{xr}}(q)$ approaches $C_{\mathrm{x} 1}(q)$. Thus, with the large- $q$ asymptotics of $I_{\mathrm{x}}(q)$ [see Equation (B.9)] and the beyond RPA-corrections analogue Equation (3.9), one obtains

$$
C_{\mathrm{x}}(q \rightarrow \infty)=g_{\mathrm{a}}(0) \frac{\omega_{\mathrm{pl}}^{2}}{q^{4}}+\left(\frac{8}{5}+c_{2}\right) \frac{\omega_{\mathrm{pl}}^{2}}{q^{6}}+\cdots,
$$

perhaps with an additional correlation parameter $c_{2}$. In comparison with Equation (3.10), the $1 / q^{6}$ tail is strongly enhanced (i.e. four times larger). Note that the difference of the prefactors is just $6 / 5=4 t_{0}$. The large- $q$ asymptotics of $C_{\mathrm{x}}(q)$ begins with the same expression as $C_{\mathrm{d}}(q)$, thus $C_{\mathrm{p}}=C_{\mathrm{d}}-C_{\mathrm{x}}$ begins with a term proportional to $1 / q^{6}$ :

$$
C_{\mathrm{p}}(q \rightarrow \infty)=\left(-4 t_{0}+c_{1}-c_{2}\right) \frac{\omega_{\mathrm{pl}}^{2}}{q^{6}}+\cdots, \quad t_{0}=\frac{3}{10},
$$

which makes $q^{2} C_{\mathrm{p}}(q)$ and even $q^{4} C_{\mathrm{p}}(q)$ integrable, see Equation (A.14). Comparison with Equation (A.13), that follows from the curvature theorem [Equation (A.11)], shows that $-4 t_{0}+c_{1}-c_{2}=-3 g_{\mathrm{p}}^{\prime \prime}(0)$ as a sum rule, hence the asymptotics (4.12) is recovered.

The asymptotics of the SFs are summarized in Table 1 and Table 2; for the high-density limit with $g_{\mathrm{a}}(0) \rightarrow 1$ and $g_{\mathrm{p}}^{\prime \prime}(0) \rightarrow 2 / 5$, the decoration drops off and the RPA limiting values reemerge. They show once more how the short-range correlation parameters determine the large- $q$ asymptotics of the SFs, what is effected by the cusp and curvature theorems. As a side result, the on-top curvature of the Fermi hole is rederived in terms 
of $S_{\mathrm{p}}$, see Equation (4.12). As it follows from Table 2 the function $\Phi(x)$, defined at the beginning, see Equation (2.3), has the asymptotics $\Phi(x \rightarrow 0)=O\left(x^{4}\right)$ and $\Phi(x \rightarrow \infty)=x-O(1 / x)$. Besides, for $r_{s} \rightarrow 0$ the inflexion point of $S(q)$ is $q_{0}=3 \omega_{\mathrm{pl}} / 2, S\left(q_{0}\right)=9 \omega_{\mathrm{pl}} / 8$. For $x$ this means $x_{0}=1$. The inflexion-point trajectory for $r_{s} \rightarrow 0$, namely $S\left(q_{0}\right)=3 q_{0} / 4$, is in agreement with the slope of the ideal-Fermi-gas term at $q \rightarrow 0$.

\section{Summary and Outlook}

The focus of this paper is on the mathematics of the HEG, which on one hand is only a marginal point in the broad realm of correlated systems lacking rigorous solutions, but on the other constitutes an archetype of an extended many-body Fermi system. The small- $q$ and large- $q$ behaviors of the cumulant structure factors $C_{\mathrm{a}, \mathrm{p}}(q)$ and the structure factors $S_{\mathrm{a}, \mathrm{p}}(q)$ as well as their normalizations are summarized in Table 1 and Table 2, respectively. In these tables, " $a$ " stands for the electron pairs with antiparallel spins and " $p$ " stands for the pairs with parallel spins. This analysis is based upon rigorous constraints such as the perfect screening sum rule (or the charge neutrality condition), the plasmon sum rule with its inflexion-point trajectory, the on-top (the zero electron-electron distance) theorems for the pair densities (cusp for " $a$ " and curvature for " $p$ ") and the "old" RPA (with the Feynman diagrams like those depicted in Figure 2(a) and Figure 3(a) giving exact results for $r_{s} \rightarrow 0$ ). They are all summarized in a way similar to the GGSB paper [15] (where $S(q)$ has been parameterized by combining analytical constraints with fitting of numerical data), but modified here under the novel cumulant point of view. This is done under the premise that the cumulant decompositions (2.4), (2.5), and (A.2) are more fundamental than the seemingly simpler decomposition $S(q)=S_{0}(q)+\Delta S(q)$ into the ideal-Fermigas component $S_{0}(q)$ and the interaction-induced remainder $\Delta S(q)$. A formal analysis is presented with the spin structure (2.10) as a decisive point, leaving the task to parameterize $C_{\mathrm{a}, \mathrm{p}}(q)$ analytically as functions of $q$ and $r_{s}$ in a manner similar to that carried out in the GGSB paper [15]. The agreement of Equation (3.5) with the results of Macke [23] and Gell-Mann/Brueckner [24] and of Equation (4.10) with the calculations of Onsager et al. [54] also confirms the expressions (3.1) for " $d r$ " and (4.5) for " $x r$ ", where " $r$ " means RPA and " $d$ " and " $x$ " mean direct and exchange Feynman diagrams, respectively, see Figure 2(a) and Figure 3(a). A complete and self-consistent RPA description needs both $C_{\mathrm{dr}}(q)$, which is available from Equation (3.3), and $C_{\mathrm{xr}}(q)$, which is actually given by a complicated exchange integral in Equation (4.7). This is needed for $S_{\mathrm{p}}(q)$ in the combination $1-F(q)-C_{\mathrm{d}}(q)+C_{\mathrm{x}}(q)$, where the Fock term $F(q)$ appears, which brings in the correlated momentum distribution $n(k)$ with such terms as $C$ (i.e. the Löwdin or idempotency parameter) and $z_{\mathrm{F}}$ (i.e. the jump of $n(k)$ at $k=1$ ), the quantity $c$ to be compensated by $C_{\mathrm{x}}(0)$. With $F=F_{0}+\Delta F$ and $S_{0}=1-F_{0}$, the final expression for $S_{\mathrm{p}}$ is $S_{\mathrm{p}}=S_{0}-C_{\mathrm{d}}+\left(C_{\mathrm{x}}-\Delta F\right)$. The combination $S_{0}-C_{\mathrm{d}}$ makes the term $\left(3 q / 4-q^{3} / 16\right)$ to have the correct prefactor $(+1-1 / 2)$, such that it disappears in $S=S_{\mathrm{a}}+S_{\mathrm{p}}$, leaving twice the half plasmon term. One may ask how the Fock term $\Delta F$, respectively the combination $C_{\mathrm{x}}-\Delta F$ influences the PD $g_{\mathrm{p}}(r)$ ?

It may be that in the present paper a deeply lying confrontation emerges, namely between 1) perturbation theory, which is linear in the Feynman diagrams, and 2) the cumulant decomposition, for which higher-order reduced density matrices in terms of products of lower-order ones are characteristic.

Going back to the starting point, which rests upon the belief that the geminals and the corresponding weights that diagonalize the cumulant 2-matrix, are the most "natural" 2-body quantities to describe an extended manybody Fermi system, establishes the direction of the future studies. If these quantities are known, then they determine the structure factors, the interaction energy $v$ and-according to [10]—also the momentum distribution $n(k)$ in terms of closed-form expressions. The next task would be to derive an effective approximate 2-body scheme (a modified 2-body Schrödinger equation or the Bethe-Salpeter equation?) from the hierarchy of contracted Schrödinger equations such that it yields the cumulant geminals and their weights.

Note added in proof: Equations (4.7) and (4.16), Figure 3, and Ref. [25] at the middle of Section 1 are related to attempts around RPA+SOSEX, see [58]-[61].

\section{Acknowledgements}

The authors thank P. Gori-Giorgi in particular for drawing their attention to the Kimball trick, and are grateful to C. Gutlé, and M. Holzmann for valuable discussions and to U. Saalmann for critically reading the manuscript. 
They acknowledge P. Fulde and the Max Planck Institute for the Physics of Complex Systems Dresden for supporting this work. One of the authors (J. C.) also acknowledges funding from NCN (Poland) under grant DEC-2012/07/B/ST4/00553.

\section{References}

[1] Fulde, P. (2012) Correlated Electrons in Quantum Matter. World Scientific, Singapore City.

[2] March, N.H. (1958) Physical Review, 110, 604.

[3] Olevano, V., Titov, A., Ladisa, M., Hämäläinen, K., Huotari, S. and Holzmann, M. (2012) Physical Review B, 86, Article ID: 195123.

[4] Holzmann, M., Bernu, B., Pierleoni, C., McMinis, J., Ceperley, D.M., Olevano, V. and Site, L.D. (2011) Physical Review Letters, 107, Article ID: 110402.

[5] Huotari, S., Soininen, J.A., Pylkkänen, T., Hämäläinen, K., Issolah, A., Titov, A., McMinis, J., Kim, J., Esler, K., Ceperley, D.M., Holzmann, M. and Olevano, V. (2010) Physical Review Letters, 105, Article ID: 086403.

[6] Nechaev, I.A. and Chulkov, E.V. (2006) Physical Review B, 73, Article ID: 165112.

[7] Gori-Giorgi, P. and Ziesche, P. (2002) Physical Review B, 66, Article ID: 235116.

[8] Ziesche, P. and Tasnádi, F. (2004) Annalen der Physik (Leipzig), 13, 124. (2004) 13, 623(E).

[9] Loos, P.-F. and Gill, P.W. (2011) Physical Review B, 84, Article ID: 033103.

[10] Ziesche, P. (2012) Physical Review A, 86, Article ID: 012508. (2014) Physical Review A, 89, 059902(E).

[11] Ziesche, P. (2010) Annals of Physics (Berlin), 522, 739.

[12] Sun, J., Perdew, J.P. and Seidl, M. (2010) Physical Review B, 81, Article ID: 085123.

[13] Gori-Giorgi, P. and Savin, A. (2006) Physical Review A, 73, Article ID: 032506.

[14] Diez Muiño, R., Nagy, I. and Echenique, P.M. (2005) Physical Review B, 72, Article ID: 075117.

[15] Gori-Giorgi, P., Sacchetti, F.and Bachelet, G.B. (2000) Physical Review B, 61, 7353. (2002) Physical Review B, 66, Article ID: 159901(E).

[16] Overhauser, A.W. (1995) Canadian Journal of Physics, 73, 683-686. http://dx.doi.org/10.1139/p95-101

[17] Coleman, A.J. and Yukalov, V.I. (2000) Reduced Density Matrices. Springer, Berlin. http://dx.doi.org/10.1007/978-3-642-58304-9

[18] Cioslowski, J. (2000) Many-Electron Densities and Reduced Density Matrices. Kluwer/Plenum, New York. http://dx.doi.org/10.1007/978-1-4615-4211-7

[19] Kong, L. and Valeev, E.F. (2011) Journal of Chemical Physics, 134, Article ID: 214109. http://dx.doi.org/10.1063/1.3596948

[20] Mazziotti, D.A. (2007) Reduced Density Matrices: With Applications to Many-Electron Atoms and Molecules. WileyBlackwell, Hoboken.

[21] Juhasz, T. and Mazziotti, D.A. (2006) Journal of Chemical Physics, 125, Article ID: 174105.

[22] Kutzelnigg, W. and Mukherjee, D. (1999) Journal of Chemical Physics, 110, 2800. http://dx.doi.org/10.1063/1.478189

[23] Macke, W. and Naturf, Z. (1950) Zeitschrift für Naturforschung, 5a, 192. ${ }^{1}$

[24] Gell-Mann, M. and Brueckner, K. (1957) Physical Review, 106, 364. http://dx.doi.org/10.1103/PhysRev.106.364

[25] Ren, X., Rinke, P., Joas, C. and Scheffler, M. (2012) Journal of Materials Science, 47, 7447-7471. http://dx.doi.org/10.1007/s10853-012-6570-4

[26] Heß elmann, A. and Andreas, A. (2011) Physical Review Letters, 106, Article ID: 093001.

[27] Hofmann, J., Barth, M. and Zwerger, W. (2013) Physical Review B, 87, Article ID: 235125.

[28] Bartók, A.B., Gillan, M.J., Manby, F.R. and Csányi, G. (2013) Physical Review B, 88, Article ID: 054104. http://dx.doi.org/10.1103/PhysRevB.88.054104

[29] Ziesche, P. and Cioslowski, J. (2005) Physica A, 356, 598-608. ${ }^{2}$ http://dx.doi.org/10.1016/j.physa.2005.04.006 ${ }^{2}$

[30] Ziesche, P. (1992) Solid State Communications, 82, 597-602. http://dx.doi.org/10.1016/0038-1098(92)90108-L

[31] Ziesche, P. (2002) International Journal of Quantum Chemistry, 90, 342-354. ${ }^{3}$

\footnotetext{
${ }^{1}$ Another approach with the same result and coining of the term "RPA" is due to D. Bohm and D. Pines (1953).

${ }^{2}$ In Equation (27) the correct prefactor is $2 \alpha r_{s} /(5 \pi)$.

${ }^{3}$ The last sentence of the Abstract should be deleted.
} 
http://dx.doi.org/10.1002/qua.969

[32] Ziesche, P. in [18], p. 33.

[33] Gutlé, C. (2006) Computer Physics Communications, 174, 836-845. http://dx.doi.org/10.1016/j.cpc.2005.12.010

[34] Pines, D. and Nozières, P. (1966) The Theory of Electron Liquids, Vol. I. Benjamin, New York.

[35] Ziesche, P. (2004) Physica Status Solidi (b), 241, 3544(I). (2005) (b) 242, 2051 (II). ${ }^{4}$

[36] Ziesche, P. (2007) Physica Status Solidi (b), 244, 2022. ${ }^{5}$

[37] Hoffman, G.G. (1992) Physical Review B, 45, 8730. http://dx.doi.org/10.1103/PhysRevB.45.8730

[38] Kimball, J.C. (1976) Physical Review B, 14, 2371. http://dx.doi.org/10.1103/PhysRevB.14.2371

[39] Geldart, D.J. (1967) Canadian Journal of Physics, 45, 3139-3161. http://dx.doi.org/10.1139/p67-260

[40] Geldart, D.J.W., Houghton, A. and Vosko, S.H. (1964) Canadian Journal of Physics, 42, 1938-1968. http://dx.doi.org/10.1139/p64-183

[41] Yasuhara, H. (1972) Solid State Communications, 11, 1481-1483. http://dx.doi.org/10.1016/0038-1098(72)90504-2

[42] Herbert, J.M. (2002) Physical Review A, 66, Article ID: 052502. http://dx.doi.org/10.1103/PhysRevA.66.052502

[43] Asgari, R., Polini, M., Davoudi, B. and Tosi, M.P. (2003) Solid State Communications, 125, 139-142. http://dx.doi.org/10.1016/S0038-1098(02)00782-2

[44] Qian, Z. (2006) Physical Review B, 73, Article ID: 035106. http://dx.doi.org/10.1103/PhysRevB.73.035106

[45] Cioslowski, J. and Ziesche, P. (2005) Physical Review B, 71, Article ID: 125105. (2005) Physical Review B, 72, Article ID: 239901 (E). http://dx.doi.org/10.1103/PhysRevB.71.125105

[46] Yasuhara, H. (1974) Physica, 78, 420-434. http://dx.doi.org/10.1016/0031-8914(74)90372-3

[47] Iwamoto, N. (1986) Physical Review A, 33, 1940.

[48] Wolfram Research Inc. (2012) Mathematica, Version 8.0. Wolfram Research Inc., Champaign.

[49] Wang, Y. and Perdew, J.P. (1991) Physical Review B, 44, 13298. http://dx.doi.org/10.1103/PhysRevB.44.13298

[50] Cioslowski, J. (2012) Journal of Chemical Physics, 136, Article ID: 044109. http://dx.doi.org/10.1063/1.3679657

[51] Kimball, J.C. (1973) Physical Review A, 7, 1648. http://dx.doi.org/10.1103/PhysRevA.7.1648

[52] Kimball, J.C. (1975) Journal of Physics A, 8, 1513. http://dx.doi.org/10.1088/0305-4470/8/9/021

[53] Yasuhara, H. and Kawazoe, Y. (1976) Physica A, 85, 416-424. http://dx.doi.org/10.1016/0378-4371(76)90060-1

[54] Onsager, L., Mittag, L. and Stephen, M.J. (1966) Annalen der Physik (Leipzig), 18, $71{ }^{6}$

[55] Glasser, M.L. (1984) Journal of Computational and Applied Mathematics, 10, 293-299. http://dx.doi.org/10.1016/0377-0427(84)90041-4

[56] Rassolov, V.A., Pople, J.A. and Ratner, M.A. (2000) Physical Review B, 62, 2232. http://dx.doi.org/10.1103/PhysRevB.62.2232

[57] Rajagopal, A.K., Kimball, J.C. and Banerjee, M. (1978) Physical Review B, 18, 2339. http://dx.doi.org/10.1103/PhysRevB.18.2339

[58] Freeman, D.L. (1977) Physical Review B, 15, 5512. http://dx.doi.org/10.1103/PhysRevB.15.5512

[59] Booth, G.H., Grüneis, A., Kresse, G. and Alavi, A. (2013) Nature, 493, 365-370. http://dx.doi.org/10.1038/nature11770

[60] Shepherd, J.J. and Grüneis, A. (2013) Physical Review Letters, 110, 22640. http://dx.doi.org/10.1103/PhysRevLett.110.226401

[61] Hummel, F. and Kresse, G. (2014) Verhandlungen der Deutschen Physikalischen Gesellschaft, Tieftemperaturphysik, 84.1.

\footnotetext{
${ }^{4}$ It is shown, that the pair-density geminals, see [16], do not satisfy the plasmon sum rule.

${ }^{5}$ In Appendix C the Macke function $I(q)$ is explicitly given. The extension to the spinpolarized electron gas is in [37].

${ }^{6}$ Here the exchange integral of the 3-dimensional HEG has been evaluated analytically. This rather herculean work was extended to the d-dimensional electron gas by M. L. Glasser [55].
} 


\section{Appendix A: The Cumulant Pair Densities and Their Cusp and Curvature Theorems}

The analogue of Equation (2.4) in the direct space is

$$
g(r)=1-\frac{1}{2} f^{2}(r)-h(r)
$$

with $f(r)=\int_{0}^{\infty} \mathrm{d}\left(k^{3}\right)(\sin k r) / k r n(k)$ being the (dimensionless) 1-matrix [and $F(q)$ of Equation (2.4) being the Fourier transform of $f^{2}(r)$ ]. Equation (2.5) corresponds to the Coulomb and Fermi holes,

$$
g_{\mathrm{a}}(r)=1-h_{\mathrm{a}}(r) \text { and } g_{\mathrm{p}}(r)=1-f^{2}(r)-h_{\mathrm{p}}(r)
$$

with the spin-summed PDs $g(r)=\left[g_{\mathrm{a}}(r)+g_{\mathrm{p}}(r)\right] / 2$ and $h(r)=\left[h_{\mathrm{a}}(r)+h_{\mathrm{p}}(r)\right] / 2$. For the qualitative behavior of $h_{\mathrm{a}, \mathrm{p}}(r)$ see Figure 11 in [7] or Figure 2 and Figure 3 in [31]. The PDs are the Fourier transformed SFs,

$$
\begin{array}{cc}
g_{\mathrm{a}}(r)-1=\int_{0}^{\infty} \mathrm{d}\left(q^{3}\right) \frac{\sin q r}{q r} S_{\mathrm{a}}(q), & h_{\mathrm{a}}(r)=\int_{0}^{\infty} \mathrm{d}\left(q^{3}\right) \frac{\sin q r}{q r} C_{\mathrm{a}}(q), \\
g_{\mathrm{p}}(r)-1=\int_{0}^{\infty} \mathrm{d}\left(q^{3}\right) \frac{\sin q r}{q r}\left[S_{\mathrm{p}}(q)-1\right], & h_{\mathrm{p}}(r)=\int_{0}^{\infty} \mathrm{d}\left(q^{3}\right) \frac{\sin q r}{q r} C_{\mathrm{p}}(q),
\end{array}
$$

and

$$
g(r)-1=\int_{0}^{\infty} \mathrm{d}\left(q^{3}\right) \frac{\sin q r}{q r} \frac{1}{2}[S(q)-1], \quad h(r)=\int_{0}^{\infty} \mathrm{d}\left(q^{3}\right) \frac{\sin q r}{q r} \frac{1}{2} C(q) .
$$

The definition of the spin-summed SFs, namely $S(q)=S_{\mathrm{a}}(q)+S_{\mathrm{p}}(q)$ and $C(q)=C_{\mathrm{a}}(q)+C_{\mathrm{p}}(q)$, brings about the factors 1/2 in Equations (A.5)-(A.7). Note also the asymmetry with respect to "a” (Coulomb hole) and "p" (Fermi hole). The conditions $S_{\mathrm{a}, \mathrm{p}}(0)=0$ fix the normalizations of $1-g_{\mathrm{a}, \mathrm{p}}(r)$, known as the perfect screening sum rule, whereas the coalescing (or the on-top) values $g_{\mathrm{a}}(0)<1$ and $g_{\mathrm{p}}(0)=0$ (as a consequence of the Pauli principle) fix the normalizations of $S_{\mathrm{a}}(q)$ and $S_{\mathrm{p}}(q)-1$, respectively, and $g_{\mathrm{p}}^{\prime \prime}(0)$ fixes the fourth moment of $S_{p}(q)$. In the following, the identities [obtained from Equations (A.3)-(A.5), respectively]

$$
\begin{gathered}
\alpha^{3} \int_{0}^{\infty} \mathrm{d}\left(r^{3}\right) \frac{1}{2} h_{\mathrm{a}}(r)=C_{\mathrm{a}}(0)=0, \quad \int_{0}^{\infty} \mathrm{d}\left(q^{3}\right) C_{\mathrm{a}}(q)=h_{\mathrm{a}}(0), \\
\alpha^{3} \int_{0}^{\infty} \mathrm{d}\left(r^{3}\right) \frac{1}{2} h_{\mathrm{p}}(r)=C_{\mathrm{p}}(0)=c, \quad \int_{0}^{\infty} \mathrm{d}\left(q^{3}\right) C_{\mathrm{p}}(q)=h_{\mathrm{p}}(0)=0,
\end{gathered}
$$

and

$$
\alpha^{3} \int_{0}^{\infty} \mathrm{d}\left(r^{3}\right) h(r)=C(0)=c, \quad \int_{0}^{\infty} \mathrm{d}\left(q^{3}\right) C(q)=2 h(0)=h_{\mathrm{a}}(0)
$$

are also used. For plots of $h_{\mathrm{a}, \mathrm{p}}(r)$ with various values of the parameter $r_{s}$ see [7], Figure 11. For the on-top (the zero electron-electron distance) curvature of the Fermi hole, it holds according to Equation (A.2) that

$$
g_{\mathrm{p}}^{\prime \prime}(0)=\frac{4}{3} t-h_{\mathrm{p}}^{\prime \prime}(0), \quad g_{\mathrm{p}}^{\prime \prime}(0)>0
$$

as the consequence of $f^{\prime \prime}(0)=-2 t / 3$. Note that $0<g_{\mathrm{a}}(0)<1$ and $g_{\mathrm{p}}(0)=g_{\mathrm{p}}^{\prime}(0)=0$.

Cusp and curvature theorems: The large- $q$ behavior of $C_{\mathrm{a}, \mathrm{p}}(q)$ is related to the electron-electron coalescence theorems (a.k.a. the cusp theorems, “a” denoting antiparallel spin pairs) [31] [51] [52] [57]

$$
g_{\mathrm{a}}^{\prime}(0)=\alpha r_{s} g_{\mathrm{a}}(0) \quad \text { or } \quad h_{\mathrm{a}}^{\prime}(0)=-\alpha r_{\mathrm{s}}\left[1-h_{\mathrm{a}}(0)\right]
$$

and the curvature theorems (with " $p$ ” standing for parallel spin pairs) [57] 


$$
g_{\mathrm{p}}^{\prime \prime \prime}(0)=\frac{3}{2} \alpha r_{\mathrm{s}} g_{\mathrm{p}}^{\prime \prime}(0) \quad \text { or } \quad h_{\mathrm{p}}^{\prime \prime \prime}(0)=\frac{3}{2} \alpha r_{\mathrm{s}}\left[h_{\mathrm{p}}^{\prime \prime}(0)-\frac{4}{3} t\right],
$$

respectively. Therefore, the short-range parameters $h_{a}(0)$ and $h_{\mathrm{p}}^{\prime \prime}(0)$ determine their derivatives $h_{\mathrm{a}}^{\prime}(0)$ and $h_{\mathrm{p}}^{\prime \prime \prime}(0)$, respectively. These theorems allow one to correct the large- $q$ behavior of the SFs and of $n(k)$ and $F(q)$ beyond RPA.

Proof of Equation (4.12): The Kimball trick is used [51] thanks to P. Gori-Giorgi. Namely, a function $\tilde{C}_{\mathrm{p}}(q)$ with the asymptotics of $\sim 1 / q^{8}$ for $q \rightarrow \infty$ is defined first through $C_{\mathrm{p}}(q)=a /\left(1+q^{2}\right)^{3}+\tilde{C}_{\mathrm{p}}(q)$. Then, from the Fourier transform (A.4) and with the help of the Mathematica software [48], one obtains

$$
h_{\mathrm{p}}(r)=a \frac{3 \pi}{16}(1+r) \mathrm{e}^{-r}+\int_{0}^{\infty} \mathrm{d}\left(q^{3}\right) \tilde{C}_{\mathrm{p}}(q) \frac{\sin q r}{q r} \Rightarrow h_{\mathrm{p}}^{\prime \prime \prime}(0)=a \frac{3 \pi}{8},
$$

from which the derivatives of $h_{\mathrm{p}}(r)$ [including $h_{\mathrm{p}}^{\prime \prime \prime}(0)=(3 \pi / 8) a$ ] follow. With the above curvature theorem (A.11), it finally results in Equation (A.9), which is equivalent with

$$
C_{\mathrm{p}}(q \rightarrow \infty)=-3 g_{\mathrm{p}}^{\prime \prime}(0) \frac{\omega_{\mathrm{pl}}^{2}}{q^{6}}+\mathrm{O}\left(\frac{1}{q^{8}}\right) .
$$

Comparing this result with Equation (3.10), allows one to conclude that the sum rule $-4 t_{0}+c_{1}-c_{2}=-3 g_{\mathrm{p}}^{\prime \prime}(0)$ holds. A side product of this analysis is

$$
\int_{0}^{\infty} \mathrm{d} q q^{4} C_{\mathrm{p}}(q)=-h_{\mathrm{p}}^{\prime \prime}(0) \Leftrightarrow \int_{0}^{\infty} \mathrm{d} q q^{4}\left[1-S_{\mathrm{p}}(q)\right]=g_{\mathrm{p}}^{\prime \prime}(0) .
$$

Proof: As an intermediate step towards the above expression for $h_{\mathrm{p}}^{\prime \prime \prime}(0)$, one derives (with the help of the Mathematica software [48]) the equations

$$
h_{\mathrm{p}}^{\prime \prime}(0)=-\frac{3 \pi}{16} a-\int_{0}^{\infty} \mathrm{d} q q^{4} c_{\mathrm{p}}(q) \text { and } \int_{0}^{\infty} \frac{\mathrm{d} q q^{4}}{\left(1+q^{2}\right)^{3}}=\frac{3 \pi}{16},
$$

which lead to the first equation of Equation (A.14). From this result, the second equation follows by means of $\int_{0}^{\infty} \mathrm{d} q q^{4} F(q)=\frac{4}{3} t$ and Equation (A.9). Remark on the sign of $h_{\mathrm{p}}^{\prime \prime}(0)$ : According to Equation (A.7), the integral $\int_{0}^{\infty} \mathrm{d} q q^{2} C_{\mathrm{p}}(q)$ has to vanish. This is because the negative asymptotics (4.16) of $C_{\mathrm{p}}(q)$ is compensated by the positive branch (4.17) for small $q$. An additional factor $q^{2}$ suppressing the latter and enhancing the former. This makes the integral $\int_{0}^{\infty} \mathrm{d} q q^{4} C_{\mathrm{p}}(q)$ negative, implying $h_{\mathrm{p}}^{\prime \prime}(0) \geq 0$. For the function $t\left(r_{\mathrm{s}}\right)$, see for example [7].

\section{Appendix B: The Particle-Hole Propagator and the Special Functions $I(q)$ and $I_{\mathrm{x}}(q)$}

The building elements of the RPA Feynman diagrams (cf. Figure 1, Figure 2(a), Figure 3(a)) are the Coulomb repulsion $v(q)=q_{c}^{2} / q^{2}$ with the coupling constant $q_{c}^{2}=3 \omega_{\mathrm{pl}}^{2}=4 \alpha r_{s} / \pi$ and the one-body Green's function of free electrons with $t(\boldsymbol{k})=k^{2} / 2$,

$$
G_{0}(k, \omega)=\frac{\Theta(k-1)}{\omega-t(\boldsymbol{k})+\mathrm{i} \delta}+\frac{\Theta(1-k)}{\omega-t(\boldsymbol{k})-\mathrm{i} \delta}, \quad \delta \rightrightarrows 0 .
$$

From $G_{0}(k, \omega)$, it follows that the particle-hole propagator $Q(q, \eta)$ within RPA, given by

$$
Q(q, \eta)=-\int \frac{\mathrm{d}^{3} k}{4 \pi} \int \frac{\mathrm{d} \omega}{2 \pi \mathrm{i}} G_{0}(k, \omega), G_{0}(|\boldsymbol{k}+\boldsymbol{q}|, \omega+\eta)
$$

results in 


$$
\begin{aligned}
& Q(q, \eta)=\int \frac{\mathrm{d}^{3} k}{4 \pi}\left[\frac{\Theta^{+}(\boldsymbol{k}, \boldsymbol{q})}{\eta-\tau(\boldsymbol{k}, \boldsymbol{q})-\mathrm{i} \delta}-\frac{\Theta^{-}(\boldsymbol{k}, \boldsymbol{q})}{\eta-\tau(\boldsymbol{k}, \boldsymbol{q})+\mathrm{i} \delta}\right], \\
& \tau(\boldsymbol{k}, \boldsymbol{q})=t(\boldsymbol{k}+\boldsymbol{q})-t(\boldsymbol{k})=q\left(k \zeta+\frac{q}{2}\right), \quad \zeta=\cos \Varangle(\boldsymbol{k}, \boldsymbol{q}) .
\end{aligned}
$$

The denominators contain the excitation energy $\tau(\boldsymbol{k}, \boldsymbol{q})$ to create a hole with the momentum $\boldsymbol{k}$ inside the Fermi sphere and a particle with the momentum $\boldsymbol{k}+\boldsymbol{q}$ outside the Fermi sphere. A complicated step function, defined by $\Theta^{ \pm}(\boldsymbol{k}, \boldsymbol{q})=1$ for $k \gtrless 1$ and $|\boldsymbol{k}+\boldsymbol{q}| \lessgtr 1$, and 0 otherwise, arises from the Pauli principle. A generalization of Equation (B.3) reads

$$
\hat{Q}_{i}(q, \eta) f\left(\boldsymbol{k}_{i}\right)=\int \frac{\mathrm{d}^{3} k_{i}}{4 \pi}\left[\frac{\Theta^{+}\left(\boldsymbol{k}_{i}, \boldsymbol{q}\right)}{\eta-\tau\left(\boldsymbol{k}_{i}, \boldsymbol{q}\right)-\mathrm{i} \delta}-\frac{\Theta^{-}\left(\boldsymbol{k}_{i}, \boldsymbol{q}\right)}{\eta-\tau\left(\boldsymbol{k}_{i}, \boldsymbol{q}\right)+\mathrm{i} \delta}\right] f\left(\boldsymbol{k}_{i}\right),
$$

defining the integral operator $\hat{Q}_{i}(q, \eta), i=1,2$.

In calculations of the cumulant SF $C_{\mathrm{d} 1}(q)$ (see Figure 2(b) with the bare Coulomb repulsion), the Macke function $I(q)$ appears via

$$
\int \frac{\mathrm{d} \eta}{2 \pi \mathrm{i}} Q^{2}(q, \eta)=\frac{2}{(4 \pi)^{2}} I(q), \quad I(q)=\int \frac{\mathrm{d}^{3} k_{1} \mathrm{~d}^{3} k_{2}}{\boldsymbol{q} \cdot\left(\boldsymbol{k}_{1}+\boldsymbol{k}_{2}+\boldsymbol{q}\right)}, \quad k_{1,2}<1,\left|\boldsymbol{k}_{1,2}+\boldsymbol{q}\right|>1 .
$$

When dealing with the corresponding exchange term $C_{\mathrm{x} 1}(q)$ (see Figure 3(b) with the bare Coulomb repulsion), one obtains by means of contour integration

$$
\left.\int \frac{\mathrm{d} \eta}{2 \pi \mathrm{i}} \hat{Q}_{1}\left(q^{\prime}, \eta\right) \hat{Q}_{2}\left(q^{\prime},-\eta\right) v\left(q^{\prime}\right)\right|_{q^{\prime}=k_{1}+\boldsymbol{k}_{2}+q}=\frac{2}{(4 \pi)^{2}} I_{\mathrm{x}}(q) v(q),
$$

where

$$
I_{\mathrm{x}}(q)=\int \frac{\mathrm{d}^{3} k_{1} \mathrm{~d}^{3} k_{2}}{\boldsymbol{q} \cdot\left(\boldsymbol{k}_{1}+\boldsymbol{k}_{2}+\boldsymbol{q}\right)} \frac{q^{2}}{\left(\boldsymbol{k}_{1}+\boldsymbol{k}_{2}+\boldsymbol{q}\right)^{2}}, \quad k_{1,2}<1, \quad\left|\boldsymbol{k}_{1,2}+\boldsymbol{q}\right|>1
$$

defines the exchange counterpart $I_{\mathrm{x}}(q)$ of the Macke function $I(q)$. The functions $I(q)$ and $I_{\mathrm{x}}(q)$ have the following properties

$$
\begin{gathered}
I(q \rightarrow 0)=\frac{8 \pi^{2}}{3}(1-\ln 2) q-\frac{\pi^{2}}{6} q^{3}+\cdots, \\
I(2)=\frac{4 \pi^{2}}{15}(13-16 \ln 2), \quad I^{\prime}(2)=-\frac{8 \pi^{2}}{5}(-1+2 \ln 2), \\
I(q \rightarrow \infty)=\left(\frac{4 \pi}{3}\right)^{2}\left(\frac{1}{q^{2}}+\frac{2}{5} \frac{1}{q^{4}}+\frac{72}{125} \frac{1}{q^{6}}+\cdots\right), \\
\int_{0}^{\infty} \mathrm{d} q I(q)=\frac{8 \pi^{2}}{45}\left(\pi^{2}+6 \ln 2-3\right),
\end{gathered}
$$

and

$$
\begin{gathered}
I_{\mathrm{x}}(q \rightarrow 0)=\pi^{2}(2 \ln 2-1) q^{3}+O(q)^{5}, \\
I_{\mathrm{x}}(2)=\left(\frac{2 \pi}{3}\right)^{2}\left(-2-\pi^{2}+20 \ln 2\right), \quad I_{\mathrm{x}}^{\prime}(2)=-\frac{2 \pi^{2}}{9}\left(8+\pi^{2}-8 \ln 2\right), \\
I_{\mathrm{x}}(q \rightarrow \infty)=\left(\frac{4 \pi}{3}\right)^{2}\left(\frac{1}{q^{2}}+\frac{8}{5} \frac{1}{q^{4}}+\cdots\right),
\end{gathered}
$$




$$
\begin{gathered}
\int_{0}^{\infty} \mathrm{d} q I_{\mathrm{x}}(q)=\int_{0}^{\infty} \mathrm{d} q I(q), \quad \int_{0}^{\infty} \mathrm{d} q \frac{I_{\mathrm{x}}(q)}{q^{2}}=\frac{4 \pi^{4}}{3}\left[\frac{1}{3} \ln 2-\frac{3}{2} \frac{\zeta(3)}{\pi^{2}}\right], \\
\int_{0}^{\infty} \mathrm{d} q q^{2}\left[I(q)-I_{\mathrm{x}}(q)\right]=-\frac{4 \pi^{2}}{3 \times 7}\left(-1+\frac{4 \pi^{2}}{5}+4 \ln 2\right),
\end{gathered}
$$

see Figure 4. $I(q)$ is known from the work of Macke [23]; it is explicitly presented e.g. in [36], where also $I^{\prime \prime}\left(2^{ \pm}\right)$is given, see Equation (C.2). $I_{x}(q)$ is known from Gutlé [33]. The original expression for $I_{x}(q)$ can be markedly simplified for $q \geq 2$, in which case

$$
\begin{aligned}
I_{\mathrm{x}}(q)= & -\frac{\pi^{2}}{72} q\left[16 \pi^{2}+16 q+16\left(3 q^{2}-32\right) \operatorname{artanh} \frac{2}{q}-96(\ln 2)^{2}+16 q\left(q^{2}-9\right) \ln \left(1-\frac{4}{q^{2}}\right)\right. \\
& +192 \ln 2 \ln \frac{q-2}{q}-3(q-2)^{2}(4+q)\left(\ln \frac{q-2}{q}\right)^{2}+6(q-4)(2+q)^{2} \ln \frac{q-2}{q} \ln \frac{2+q}{q} \\
& \left.-3(q-4)(2+q)^{2}\left(\ln \frac{2+q}{q}\right)^{2}-192 \mathrm{Li}_{2}\left(\frac{1}{2}-\frac{1}{q}\right)\right] .
\end{aligned}
$$

The small- $q$ behavior $I(q) \sim q$ causes the "Heisenberg divergence". The asymptotics $I_{\mathrm{x}}(q) \sim q^{3}$ ensures finiteness of the integral $\int_{0}^{\infty} \mathrm{d} q I_{\mathrm{x}}(q) / q^{2}$, which has been congenially calculated by Onsager et al. [54], see Equation (4.10). The flattening in the small- $q$ region, observed upon going from $I(q)$ to $I_{\mathrm{x}}(q)$, together with the "area conservation" $\int_{0}^{\infty} \mathrm{d} q\left[I(q)-I_{\mathrm{x}}(q)\right]=0$, is partly compensated by the heavy enhancement of the $1 / q^{4}$ tail (from 2/5 to 8/5), see Figure 4. Both $I(q)$ and $I_{\mathrm{x}}(q)$ consist of two branches, which encounter each other at the Fermi edge with transition momenta $|\boldsymbol{q}|=2$. They have a simple analytical behavior for $q \rightarrow 0$ and $q \rightarrow \infty$, but "in between", namely at $q=2$ it is

$$
I(2+p)=I(2)+I^{\prime}(2) p+\frac{\pi^{2}}{15}\left[a_{ \pm}+b_{ \pm}|p| \ln |p|\right] p^{2}+\cdots
$$

with $a_{+}=8+4 \ln 2, b_{+}=5$ for $q>2$ and $a_{-}=-7+4 \ln 2, b_{-}=1 / 4$ for $q<2$. For $I_{\mathrm{x}}(q)$ it similarly holds

$$
I_{\mathrm{x}}(2+p)=a+b p+c p^{2}+d p^{2} \ln p+e p^{2}(\ln p)^{2}+\cdots
$$

with (for $p>0$ )

$$
\begin{aligned}
& a=\left(\frac{2 \pi}{3}\right)^{2}\left(-2-\pi^{2}+20 \ln 2\right), \quad b=-\frac{2 \pi^{2}}{9}\left(8+\pi^{2}-8 \ln 2\right), \\
& c=\frac{\pi^{2}}{3}\left[\frac{5}{4}-11 \ln 2+6(\ln 2)^{2}\right], \quad d=-\frac{\pi^{2}}{3}\left(\frac{9}{2}+6 \ln 2\right), \quad e=\frac{\pi^{2}}{2} .
\end{aligned}
$$

The analog case $p<0$ is not known so far. The peculiar behavior at $q \approx 2$ reminds of the behavior of $n(k)$ near $k \approx 1$, see [7], Equations (6) and (7).

Remark on the types: Note that indices like pl, a, p, d, x, dr, xr are to be represented by upright types, not by italics as this is falsely partly the case e.g. in Table 1 and Table 2. 
Scientific Research Publishing (SCIRP) is one of the largest Open Access journal publishers. It is currently publishing more than 200 open access, online, peer-reviewed journals covering a wide range of academic disciplines. SCIRP serves the worldwide academic communities and contributes to the progress and application of science with its publication.

Other selected journals from SCIRP are listed as below. Submit your manuscript to us via either submit@scirp.org or Online Submission Portal.
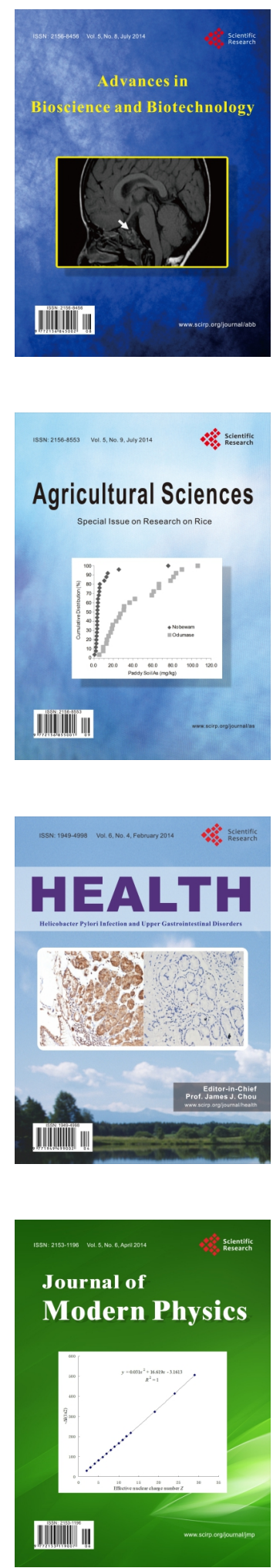
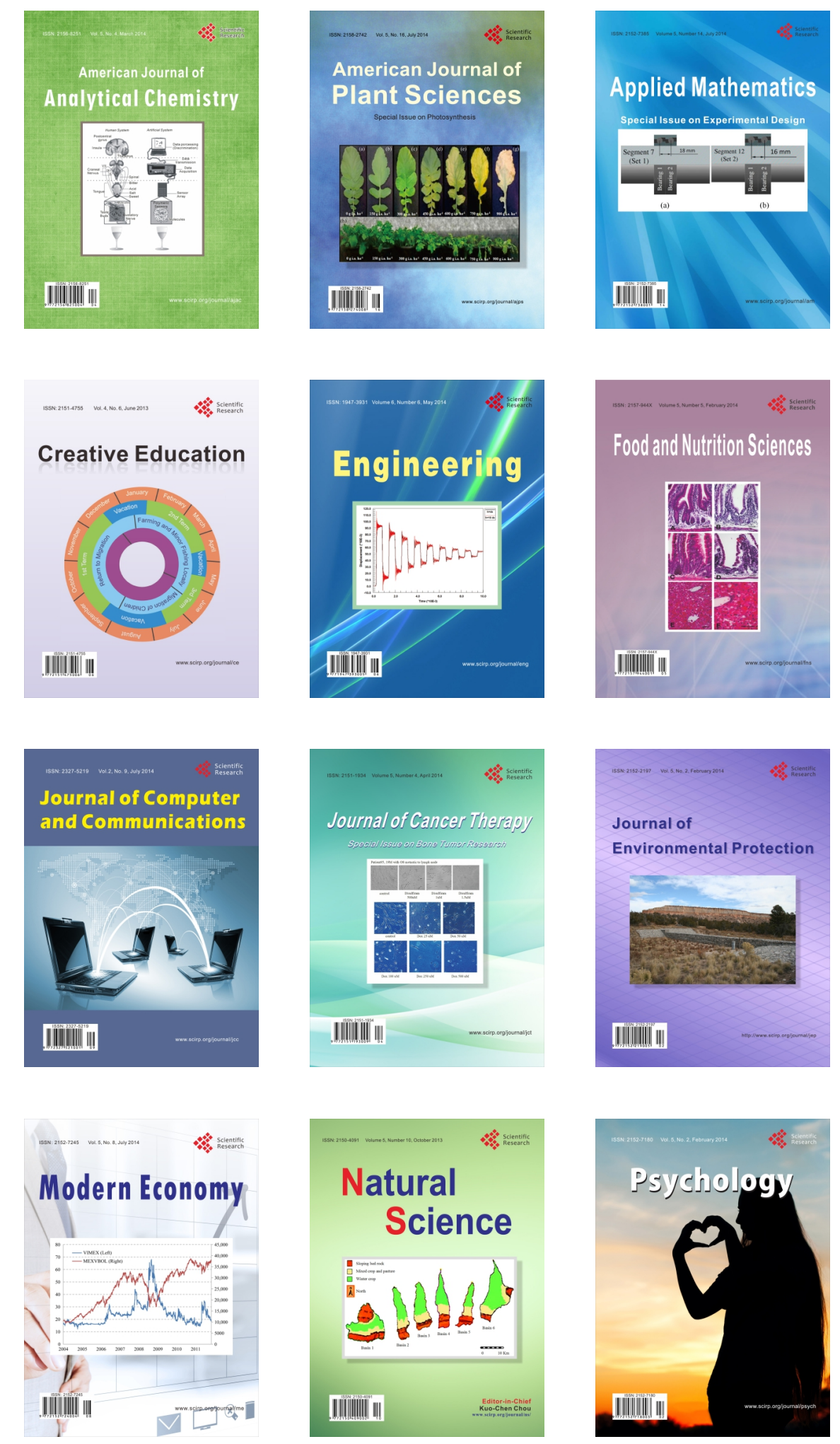